Key Words:

Tank Closure,

Chemical

Cleaning, Oxalic

Acid

Retention:

Permanent

\title{
Analysis of the Tank 5F Feed and Bleed Residual Solids
}

\author{
Michael R. Poirier \\ David P. DiPrete \\ Charles J. Coleman \\ A. L. Washington
}

July 8, 2011

Savannah River National Laboratory

Savannah River Nuclear Solutions

Aiken, SC 29808

Prepared for the U.S. Department of Energy Under

Contract Number DE-AC09-08SR22470

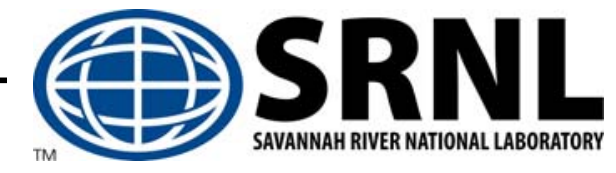




\section{DISCLAIMER}

This work was prepared under an agreement with and funded by the U.S. Government. Neither the U. S. Government or its employees, nor any of its contractors, subcontractors or their employees, makes any express or implied:

1. warranty or assumes any legal liability for the accuracy, completeness, or for the use or results of such use of any information, product, or process disclosed; or

2. representation that such use or results of such use would not infringe privately owned rights; or

3. endorsement or recommendation of any specifically identified commercial product, process, or service.

Any views and opinions of authors expressed in this work do not necessarily state or reflect those of the United States Government, or its contractors, or subcontractors.

Printed in the United States of America

Prepared for

U.S. Department of Energy 
Key Words:

Tank Closure,

Chemical

Cleaning, Oxalic

Acid

Retention:

Permanent

\title{
Analysis of the Tank 5F Feed and Bleed Residual Solids
}

\author{
Michael R. Poirier \\ David P. DiPrete \\ Charles J. Coleman \\ A. L. Washington
}

July 8, 2011

Savannah River National Laboratory

Savannah River Nuclear Solutions

Savannah River Site

Aiken, SC 29808 


\section{REVIEWS AND APPROVALS}

\section{Authors}

M. R. Poirier, SRNL, Advanced Characterization \& Processing

Date

D. P. DiPrete, SRNL, Analytical Development

Date

C. J. Coleman, SRNL, Analytical Development

Date

A. L. Washington, SRNL, Advanced Characterization \& Processing

Date

\section{Design Check}

M. S. Hay, SRNL, Advanced Characterization \& Processing

Date

\section{Management}

F. M. Pennebaker,, Manager, SRNL,

Date

Advanced Characterization \& Processing

S. L. Marra, Manager, SRNL E\&CPT Research Programs

Date

\section{Customer}

W. L. Isom, Manager, SRR Engineering

Date 


\section{TABLE OF CONTENTS}

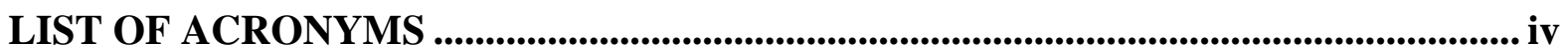

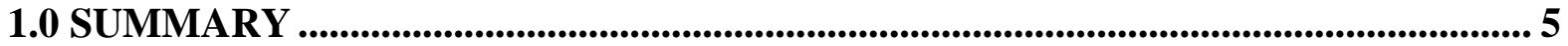

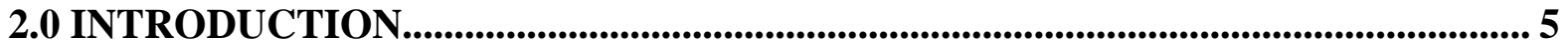

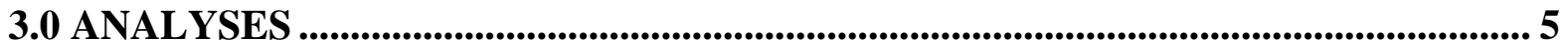

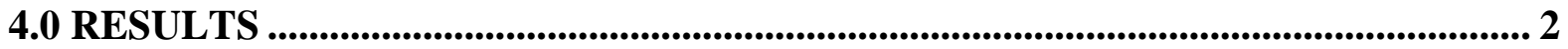

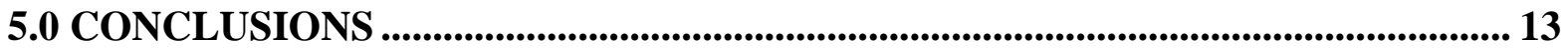

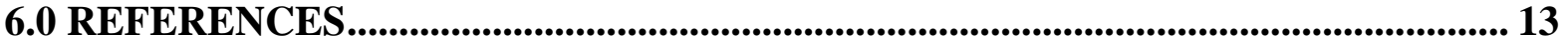




\section{LIST OF ACRONYMS}

$\begin{array}{ll}\text { LWO } & \text { Liquid Waste Organization } \\ \text { SMP } & \text { Submersible Mixer Pumps } \\ \text { SRNL } & \text { Savannah River National Laboratory } \\ \text { SRR } & \text { Savannah River Remediation } \\ \text { SRS } & \text { Savannah River Site }\end{array}$




\subsection{SUMMARY}

Savannah River Remediation (SRR) is preparing Tank 5F for closure. As part of Tank 5F Closure Mechanical Cleaning, SRR conducted a "Feed and Bleed" process in Tank 5F. Following this "Feed and Bleed” Mechanical Cleaning in Tank 5F, SRR collected two tank heel samples (referred to as sample 1 and sample 2) under Riser 5 to determine the composition of the material remaining in the tanks. This document describes sample analysis results.

The conclusions from this analysis follow.

- The anions measured all had a concentration less than $250 \mathrm{mg} / \mathrm{kg}$, except for oxalate, which had a concentration of $2100-2400 \mathrm{mg} / \mathrm{kg}$.

- The measured cations with the highest concentration were iron $(432,000-519,000$ $\mathrm{mg} / \mathrm{kg}$ ), nickel (54,600 - 69,300 mg/kg), and manganese (35,200 - 42,100 mg/kg). All other cations measured less than 13,000 mg/kg.

- The radionuclides present in the highest concentration are ${ }^{90} \mathrm{Sr}\left(3.0 \times 10^{10} \mathrm{dpm} / \mathrm{g}\right),{ }^{137} \mathrm{Cs}$ (6.8 $\left.\times 10^{8} \mathrm{dpm} / \mathrm{g}\right)$, and ${ }^{241} \mathrm{Am}\left(1.4 \times 10^{8}-1.8 \times 10^{8} \mathrm{dpm} / \mathrm{g}\right)$.

- The particle size analysis shows a large fraction of particles greater than $100 \mu$.

\subsection{INTRODUCTION}

Savannah River Remediation (SRR) is preparing Tank 5F for closure. Part of tank closure is mechanical sludge removal.

Following Phase II Mechanical Cleaning (i.e., "Feed and Bleed”) in Tank 5F, SRR collected two tank heel samples (referred to as sample 1 and sample 2) on April 8, 2010 from the solids accumulation under Riser 5 to determine the composition of the material remaining in the tanks. These samples were collected using a scraper sampling tool that collected approximately 20 grams of material per scrape. This document describes sample analysis results. The results include anions, cations, ${ }^{60} \mathrm{Co},{ }^{90} \mathrm{Sr},{ }^{99} \mathrm{Tc},{ }^{137} \mathrm{Cs},{ }^{154} \mathrm{Eu},{ }^{233} \mathrm{U},{ }^{234} \mathrm{U},{ }^{235} \mathrm{U},{ }^{236} \mathrm{U},{ }^{238} \mathrm{U},{ }^{237} \mathrm{~Np},{ }^{238} \mathrm{Pu}$, ${ }^{239} \mathrm{Pu},{ }^{239 / 240} \mathrm{Pu},{ }^{240} \mathrm{Pu}{ }^{241} \mathrm{Pu},{ }^{244} \mathrm{Pu},{ }^{241} \mathrm{Am},{ }^{243} \mathrm{Am},{ }^{242 \mathrm{~m}} \mathrm{Am},{ }^{243} \mathrm{Cm},{ }^{245} \mathrm{Cm},{ }^{247} \mathrm{Cm},{ }^{249} \mathrm{Cf},{ }^{251} \mathrm{Cf}$, ${ }^{242} \mathrm{Cm},{ }^{244} \mathrm{Cm}$, and particle size. The purpose of the document is to provide early characterization data for use in the evaluation of the heel removal process in Tank $5 \mathrm{~F}$ and to support planning for future heel removal efforts. ${ }^{1,2}$

\subsection{ANALYSES}

SRNL received two solid replicate scrape samples from under riser 5 in Tank 5F (see Figure 1). The sample contained no free liquid. SRNL prepared four subsamples from each of the samples. In addition, we prepared one blank sample (using the digestion reagents only). From each subsample, personnel took a sample for digestion by aqua regia, a sample for digestion by sodium peroxide fusion, and a sample for contact wit deionized water. The aqua regia digestion consists of heating the sample at $115^{\circ} \mathrm{C}$ for two hours with a 3:1 mixture of concentrated hydrochloric acid and concentrated nitric acid in a sealed Teflon pressure. The sodium peroxide fusion consists of heating the sample at $675^{\circ} \mathrm{C}$ for 10 minutes with sodium peroxide in a zirconium crucible followed by dissolution of the fusion residue with nitric acid. The water 
contact sample was prepared by contacting approximately 0.5 grams of sample with approximately 25 grams of deionized water.

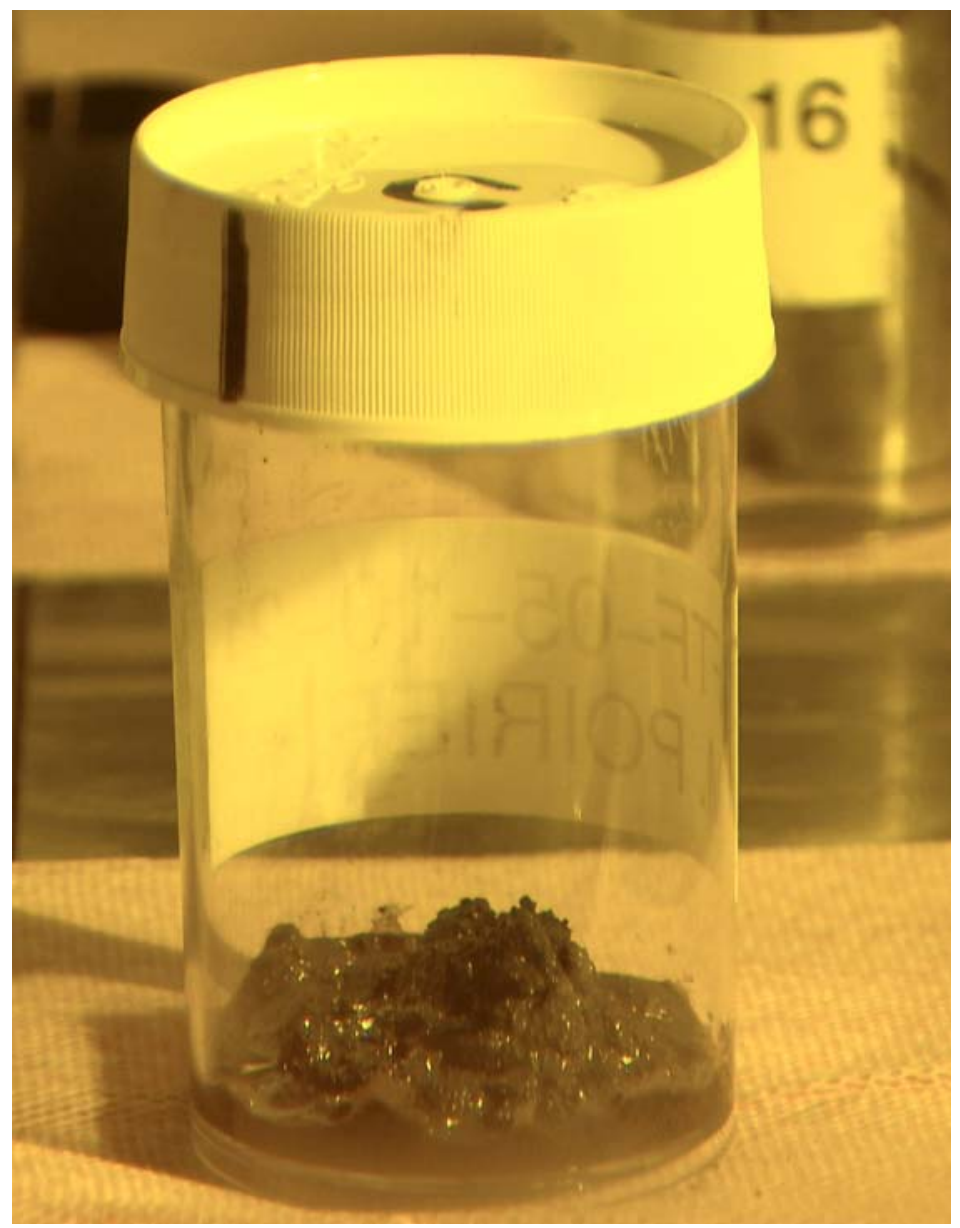

Figure 1. Tank 5F Feed and Bleed sample

The samples were analyzed for cations (ICPES), anions (IC), mercury (by Cold Vapor Atomic Absorption, CVAA, spectroscopy), and radionuclides (ICPMS, liquid scintillation counting, gamma scan, PuTTA, ${ }^{90} \mathrm{Sr},{ }^{99} \mathrm{Tc}$, and Am/Cm methods).

\subsection{RESULTS}

Table 1 shows the anion composition of sample 1, and Table 2 shows the anion composition of sample 2. The samples were prepared by performing a water leach of the solids. The concentrations of all species are less than $250 \mu \mathrm{g} / \mathrm{g}$, except for oxalate that had a concentration of $2100-2400 \mu \mathrm{g} / \mathrm{g}$. Because the solid particles were contacted with water rather than nitric acid, the actual oxalate concentration in the sludge may be higher. 
Table 1. Sample 1 Anion Analysis

$\begin{array}{lllllll} & \begin{array}{l}300271114 \\ \text { Blank } \\ (\mu \mathrm{g} / \mathrm{g})\end{array} & \begin{array}{l}300271106 \\ \text { Prelim } 1 \\ (\mu \mathrm{g} / \mathrm{g})\end{array} & \begin{array}{l}300271107 \\ \text { Prelim } 2 \\ (\mu \mathrm{g} / \mathrm{g})\end{array} & \begin{array}{l}300271108 \\ \text { Prelim } 3 \\ (\mu \mathrm{g} / \mathrm{g})\end{array} & \begin{array}{l}\text { 300271109 } \\ \text { Prelim } 4 \\ (\mu \mathrm{g} / \mathrm{g})\end{array} & \begin{array}{l}\text { Average } \\ (\mu \mathrm{g} / \mathrm{g})\end{array} \\ \begin{array}{l}\text { Analyte } \\ \begin{array}{l}\text { Fluoride } \\ \left(\mathrm{F}^{-}\right)\end{array}\end{array} & <50 & <50 & <45 & <48 & <50 & <48 \\ \begin{array}{l}\text { Formate } \\ \left(\mathrm{HCOO}^{-}\right)\end{array} & <50 & 50 & 45 & 48 & 50 & 48 \\ \begin{array}{l}\text { Chloride } \\ \left(\mathrm{Cl}^{-}\right)\end{array} & 100 & 100 & 90 & 97 & 100 & 97 \\ \begin{array}{l}\text { Nitrite } \\ \left(\mathrm{NO}_{2}{ }^{-}\right)\end{array} & <50 & <50 & <45 & <48 & <50 & <48 \\ \begin{array}{l}\text { Nitrate } \\ \left(\mathrm{NO}_{3}{ }^{-}\right)\end{array} & 100 & 150 & 90 & 97 & 100 & 110 \\ \begin{array}{l}\text { Phosphate } \\ \left(\mathrm{PO}_{4}{ }^{3-}\right)\end{array} & <250 & <250 & <220 & <240 & <250 & <240 \\ \begin{array}{l}\text { Sulfate } \\ \left(\mathrm{SO}_{4}{ }^{2-}\right)\end{array} & 100 & 150 & 180 & 150 & 150 & 160 \\ \begin{array}{l}\text { Oxalate } \\ \left(\mathrm{C}_{2} \mathrm{O}_{4}{ }^{2-}\right)\end{array} & <50 & 2300 & 2100 & 2100 & 2100 & 2150 \\ \begin{array}{l}\text { Bromide } \\ \left(\mathrm{Br}^{-}\right)\end{array} & <50 & <50 & <45 & <48 & <50 & <48\end{array}$

Table 2. Sample 2 Anion Analysis

\begin{tabular}{|c|c|c|c|c|c|c|}
\hline Analyte & $\begin{array}{l}300271114 \\
\text { Blank } \\
(\mu g / g)\end{array}$ & $\begin{array}{l}300271110 \\
\text { Prelim } 5 \\
(\mu \mathrm{g} / \mathrm{g})\end{array}$ & $\begin{array}{l}300271111 \\
\text { Prelim } 6 \\
(\mu \mathrm{g} / \mathrm{g})\end{array}$ & $\begin{array}{l}30027112 \\
2 \text { Prelim } 7 \\
(\mu \mathrm{g} / \mathrm{g})\end{array}$ & $\begin{array}{l}300271113 \\
\text { Prelim } 8 \\
(\mu \mathrm{g} / \mathrm{g})\end{array}$ & $\begin{array}{l}\text { Average } \\
(\mu g / g)\end{array}$ \\
\hline $\begin{array}{l}\text { Fluoride } \\
\left(F^{-}\right)\end{array}$ & $<50$ & $<42$ & $<41$ & $<41$ & $<42$ & $<42$ \\
\hline $\begin{array}{l}\text { Formate } \\
\left(\mathrm{HCOO}^{-}\right)\end{array}$ & $<50$ & 42 & 41 & 41 & 42 & 42 \\
\hline $\begin{array}{l}\text { Chloride } \\
\left(\mathrm{Cl}^{-}\right)\end{array}$ & 100 & 83 & 81 & 83 & 84 & 83 \\
\hline $\begin{array}{l}\text { Nitrite } \\
\left.\left(\mathrm{NO}_{2}^{-}\right)^{-}\right)\end{array}$ & $<50$ & $<42$ & $<41$ & $<41$ & $<42$ & $<42$ \\
\hline $\begin{array}{l}\text { Nitrate } \\
\left(\mathrm{NO}_{3}{ }^{-}\right)\end{array}$ & 100 & 83 & 81 & 83 & 84 & 83 \\
\hline $\begin{array}{l}\text { Phosphate } \\
\left(\mathrm{PO}_{4}{ }^{3-}\right)\end{array}$ & $<250$ & $<210$ & $<200$ & $<210$ & $<210$ & $<210$ \\
\hline $\begin{array}{l}\text { Sulfate } \\
\left(\mathrm{SO}_{4}{ }^{2-}\right)\end{array}$ & 100 & 250 & 240 & 250 & 250 & 250 \\
\hline $\begin{array}{l}\text { Oxalate } \\
\left(\mathrm{C}_{2} \mathrm{O}_{4}{ }^{2-}\right)\end{array}$ & $<50$ & 2300 & 2400 & 2400 & 2400 & 2400 \\
\hline $\begin{array}{l}\text { Bromide } \\
\left(\mathrm{Br}^{-}\right)\end{array}$ & $<50$ & $<42$ & $<41$ & $<41$ & $<42$ & $<41$ \\
\hline
\end{tabular}


Table 3 and Table 4 show the cation composition for sample 1 and sample 2, respectively. The samples were prepared by performing either an aqua regia digestion or a peroxide fusion digestion. The species with the largest concentration are iron $(432,000-519,000 \mathrm{mg} / \mathrm{kg})$, nickel (54,600 - 69,300 mg/kg), manganese (35,200 - 42,100 mg/kg), uranium (9,160 - 12,100 mg/kg), silicon $(7,790-9,580 \mathrm{mg} / \mathrm{kg})$, aluminum $(4,520-5,510 \mathrm{mg} / \mathrm{kg})$, cerium $(3,290-4,450 \mathrm{mg} / \mathrm{kg})$, calcium (2,310 - 3,140 mg/kg), mercury (2,010 - 2,090 mg/kg), barium (1,970 - 2,370 mg/kg), and lanthanum $(1,250-1,820 \mathrm{mg} / \mathrm{kg})$. Since the calcium concentration in the blanks was approximately the same as the calcium concentration in the samples, the calcium may be from the dissolution agents.

Table 3. Sample 1 Cation Analysis

\begin{tabular}{|c|c|c|c|c|c|c|c|}
\hline & $300271087^{a}$ & $300271079^{b}$ & $300271080^{c}$ & $300271081^{d}$ & $300271082^{\mathrm{e}}$ & & \\
\hline Analyte & $\begin{array}{l}\text { Blank } \\
(\mu \mathrm{g} / \mathrm{mL})\end{array}$ & $\begin{array}{l}\text { Prelim } 1 \\
(\mu \mathrm{g} / \mathrm{g})\end{array}$ & $\begin{array}{l}\text { Prelim } 2 \\
(\mu \mathrm{g} / \mathrm{g})\end{array}$ & $\begin{array}{l}\text { Prelim } 3 \\
(\mu \mathrm{g} / \mathrm{g})\end{array}$ & $\begin{array}{l}\text { Prelim } 4 \\
(\mu \mathrm{g} / \mathrm{g})\end{array}$ & $\begin{array}{l}\text { Average } \\
(\mu \mathrm{g} / \mathrm{g})\end{array}$ & $\begin{array}{l}\text { Uncertainty } \\
(\mu \mathrm{g} / \mathrm{g})\end{array}$ \\
\hline $\mathrm{Ag}$ & $<2.57 \mathrm{E}+02$ & $<2.25 \mathrm{E}+02$ & $<2.15 \mathrm{E}+02$ & $<2.43 \mathrm{E}+02$ & $<2.31 \mathrm{E}+02$ & $<2.29 \mathrm{E}+02$ & - \\
\hline $\mathrm{Al}$ & $8.20 \mathrm{E}+02$ & $4.68 \mathrm{E}+03$ & $4.88 \mathrm{E}+03$ & $5.37 E+03$ & $5.51 \mathrm{E}+03$ & $5.11 \mathrm{E}+03$ & $3.94 \mathrm{E}+02$ \\
\hline As & $<5.50 \mathrm{E}+00$ & $<3.63 \mathrm{E}+00$ & $<5.08 \mathrm{E}+00$ & $<4.30 \mathrm{E}+00$ & $<5.27 \mathrm{E}+00$ & $<4.57 \mathrm{E}+00$ & - \\
\hline B & $<3.00 \mathrm{E}+02$ & $<2.63 \mathrm{E}+02$ & $<2.51 \mathrm{E}+02$ & $<2.84 \mathrm{E}+02$ & $<2.70 \mathrm{E}+02$ & $<2.67 \mathrm{E}+02$ & - \\
\hline $\mathrm{Ba}$ & $<9.44 \mathrm{E}+01$ & $1.97 \mathrm{E}+03$ & $2.05 \mathrm{E}+03$ & $2.34 \mathrm{E}+03$ & $2.37 \mathrm{E}+03$ & $2.18 \mathrm{E}+03$ & $2.02 E+02$ \\
\hline $\mathrm{Be}$ & $<5.60 \mathrm{E}+01$ & $<4.90 \mathrm{E}+01$ & $<4.68 \mathrm{E}+01$ & $<5.29 \mathrm{E}+01$ & $<5.04 \mathrm{E}+01$ & $<4.98 \mathrm{E}+01$ & - \\
\hline $\mathrm{Ca}$ & $1.90 \mathrm{E}+03$ & $2.31 E+03$ & $2.45 \mathrm{E}+03$ & $2.41 \mathrm{E}+03$ & $2.94 \mathrm{E}+03$ & $2.53 \mathrm{E}+03$ & $2.81 \mathrm{E}+02$ \\
\hline $\mathrm{Cd}$ & $<1.22 \mathrm{E}+02$ & $<1.08 \mathrm{E}+02$ & $<1.03 \mathrm{E}+02$ & $<1.16 \mathrm{E}+02$ & $<1.11 \mathrm{E}+02$ & $<1.10 \mathrm{E}+02$ & \\
\hline $\mathrm{Ce}$ & $<5.28 \mathrm{E}+02$ & $3.29 E+03$ & $3.46 \mathrm{E}+03$ & 3.97E+03 & $3.93 E+03$ & $3.66 \mathrm{E}+03$ & $3.40 \mathrm{E}+02$ \\
\hline Co & $<6.80 \mathrm{E}+01$ & $2.06 \mathrm{E}+02$ & $2.02 \mathrm{E}+02$ & $2.43 E+02$ & $2.59 \mathrm{E}+02$ & $2.28 \mathrm{E}+02$ & $2.80 \mathrm{E}+01$ \\
\hline $\mathrm{Cr}$ & $<8.40 \mathrm{E}+01$ & $3.00 \mathrm{E}+02$ & $3.25 E+02$ & $3.97 \mathrm{E}+02$ & $3.93 \mathrm{E}+02$ & $3.54 \mathrm{E}+02$ & $4.87 E+01$ \\
\hline $\mathrm{Cu}$ & $<1.14 \mathrm{E}+02$ & $7.11 \mathrm{E}+02$ & $6.82 \mathrm{E}+02$ & $8.10 \mathrm{E}+02$ & $7.96 \mathrm{E}+02$ & $7.50 \mathrm{E}+02$ & $6.29 \mathrm{E}+01$ \\
\hline $\mathrm{Fe}$ & $1.71 \mathrm{E}+02$ & $4.36 \mathrm{E}+05$ & $4.32 \mathrm{E}+05$ & $5.19 \mathrm{E}+05$ & $5.13 \mathrm{E}+05$ & $4.75 \mathrm{E}+05$ & $4.74 \mathrm{E}+04$ \\
\hline Gd & $<3.40 \mathrm{E}+02$ & $<2.96 \mathrm{E}+02$ & $<2.84 \mathrm{E}+02$ & $<3.20 \mathrm{E}+02$ & $<3.06 \mathrm{E}+02$ & $<3.02 \mathrm{E}+02$ & - \\
\hline $\mathrm{Hg}$ & $<2.20 \mathrm{E}+01$ & $2.05 E+03$ & $2.05 \mathrm{E}+03$ & $2.06 \mathrm{E}+03$ & $2.13 \mathrm{E}+03$ & $2.07 \mathrm{E}+03$ & $3.86 \mathrm{E}+01$ \\
\hline $\mathrm{K}$ & $<2.79 \mathrm{E}+03$ & $<2.44 \mathrm{E}+03$ & $<2.35 \mathrm{E}+03$ & $<2.64 \mathrm{E}+03$ & $<2.51 \mathrm{E}+03$ & $<2.49 \mathrm{E}+03$ & - \\
\hline $\mathrm{La}$ & $<8.64 \mathrm{E}+01$ & $1.25 \mathrm{E}+03$ & $1.29 \mathrm{E}+03$ & $1.49 \mathrm{E}+03$ & $1.47 \mathrm{E}+03$ & $1.38 \mathrm{E}+03$ & $1.23 \mathrm{E}+02$ \\
\hline $\mathrm{Li}$ & $<1.38 \mathrm{E}+02$ & $4.98 E+02$ & $5.11 \mathrm{E}+02$ & $5.65 \mathrm{E}+02$ & $5.78 \mathrm{E}+02$ & $5.38 \mathrm{E}+02$ & $3.94 \mathrm{E}+01$ \\
\hline $\mathrm{Mg}$ & $<1.76 \mathrm{E}+01$ & $4.11 E+02$ & $4.47 \mathrm{E}+02$ & $5.19 \mathrm{E}+02$ & $5.44 \mathrm{E}+02$ & $4.80 \mathrm{E}+02$ & $6.18 \mathrm{E}+01$ \\
\hline $\mathrm{Mn}$ & $<1.60 \mathrm{E}+01$ & $3.53 \mathrm{E}+04$ & $3.65 \mathrm{E}+04$ & $4.13 \mathrm{E}+04$ & $4.21 \mathrm{E}+04$ & $3.88 \mathrm{E}+04$ & $3.40 \mathrm{E}+03$ \\
\hline Mo & $<5.01 \mathrm{E}+02$ & $<4.38 \mathrm{E}+02$ & $<4.18 \mathrm{E}+02$ & $<4.73 \mathrm{E}+02$ & $<4.51 \mathrm{E}+02$ & $<4.45 \mathrm{E}+02$ & - \\
\hline $\mathrm{Ni}$ & $<1.88 \mathrm{E}+02$ & $5.46 \mathrm{E}+04$ & $5.67 \mathrm{E}+04$ & $6.40 \mathrm{E}+04$ & $6.46 \mathrm{E}+04$ & $6.00 \mathrm{E}+04$ & $5.07 E+03$ \\
\hline $\mathrm{P}$ & $<1.35 \mathrm{E}+03$ & $<1.18 \mathrm{E}+03$ & $<1.13 \mathrm{E}+03$ & $<1.28 \mathrm{E}+03$ & $<1.22 \mathrm{E}+03$ & $<1.20 \mathrm{E}+03$ & - \\
\hline $\mathrm{Pb}$ & $<5.85 \mathrm{E}+02$ & $8.20 \mathrm{E}+02$ & $8.26 \mathrm{E}+02$ & $7.81 \mathrm{E}+02$ & $9.60 \mathrm{E}+02$ & $8.47 \mathrm{E}+02$ & $7.81 \mathrm{E}+01$ \\
\hline S & $<6.00 \mathrm{E}+03$ & $<5.25 \mathrm{E}+03$ & $<5.01 \mathrm{E}+03$ & $<5.67 \mathrm{E}+03$ & $<5.40 \mathrm{E}+03$ & $<5.33 \mathrm{E}+03$ & - \\
\hline $\mathrm{Sb}$ & $<5.50 \mathrm{E}+02$ & $<4.82 \mathrm{E}+02$ & $<4.60 \mathrm{E}+02$ & $<5.20 \mathrm{E}+02$ & $<4.95 \mathrm{E}+02$ & $<4.89 \mathrm{E}+02$ & - \\
\hline Se & $<1.10 \mathrm{E}+01$ & $<7.27 \mathrm{E}+00$ & $<1.02 \mathrm{E}+01$ & $<8.59 \mathrm{E}+00$ & $<1.05 \mathrm{E}+01$ & $<9.14 \mathrm{E}+00$ & - \\
\hline $\mathrm{Si}$ & $<1.85 \mathrm{E}+02$ & $7.79 \mathrm{E}+03$ & $8.24 \mathrm{E}+03$ & $9.16 \mathrm{E}+03$ & $9.46 \mathrm{E}+03$ & $8.66 \mathrm{E}+03$ & $7.80 \mathrm{E}+02$ \\
\hline Sn & $<5.15 \mathrm{E}+02$ & $<4.50 \mathrm{E}+02$ & $<4.31 \mathrm{E}+02$ & $<4.86 \mathrm{E}+02$ & $<4.64 \mathrm{E}+02$ & $<4.58 \mathrm{E}+02$ & - \\
\hline $\mathrm{Sr}$ & $<2.00 \mathrm{E}+01$ & $3.30 \mathrm{E}+02$ & $3.36 \mathrm{E}+02$ & $3.97 \mathrm{E}+02$ & $3.96 \mathrm{E}+02$ & $3.65 E+02$ & $3.67 \mathrm{E}+01$ \\
\hline $\mathrm{Ti}$ & $<1.36 \mathrm{E}+01$ & $2.42 \mathrm{E}+02$ & $2.51 E+02$ & $2.86 \mathrm{E}+02$ & $2.84 \mathrm{E}+02$ & $2.66 \mathrm{E}+02$ & $2.25 \mathrm{E}+01$ \\
\hline $\mathrm{U}$ & $<6.95 \mathrm{E}+03$ & $9.16 \mathrm{E}+03$ & $9.21 \mathrm{E}+03$ & $1.04 \mathrm{E}+04$ & $1.20 \mathrm{E}+04$ & $1.02 \mathrm{E}+04$ & $1.33 \mathrm{E}+03$ \\
\hline V & $<8.32 \mathrm{E}+01$ & $<7.28 \mathrm{E}+01$ & $<6.94 \mathrm{E}+01$ & $<7.86 \mathrm{E}+01$ & $<7.48 \mathrm{E}+01$ & $<7.39 \mathrm{E}+01$ & - \\
\hline $\mathrm{Zn}$ & $<1.21 \mathrm{E}+02$ & $3.66 \mathrm{E}+02$ & $3.94 \mathrm{E}+02$ & $4.50 \mathrm{E}+02$ & $4.43 \mathrm{E}+02$ & $4.13 \mathrm{E}+02$ & $4.02 \mathrm{E}+01$ \\
\hline
\end{tabular}

\footnotetext{
${ }^{a}$ Arsenic, mercury, and selenium obtained from LIMS number 300271096.

${ }^{\mathrm{b}}$ Arsenic, mercury, and selenium obtained from LIMS number 300271088.

c Arsenic, mercury, and selenium obtained from LIMS number 300271089.

d Arsenic, mercury, and selenium obtained from LIMS number 300271090.

e Arsenic, mercury, and selenium obtained from LIMS number 300271091.
} 
SRNL-STI-2011-00167, REV. 0

Table 4. Sample 2 Cation Analysis

\begin{tabular}{|c|c|c|c|c|c|c|c|}
\hline & $300271087^{f}$ & $300271083^{g}$ & $300271084^{\mathrm{h}}$ & $300271085^{i}$ & $300271086^{j}$ & & \\
\hline Analyte & $\begin{array}{l}\text { Blank } \\
(\mu \mathrm{g} / \mathrm{g})\end{array}$ & $\begin{array}{l}\text { Prelim } 5 \\
(\mu g / g)\end{array}$ & $\begin{array}{l}\text { Prelim } 6 \\
(\mu \mathrm{g} / \mathrm{g})\end{array}$ & $\begin{array}{l}\text { Prelim } 7 \\
(\mu g / g)\end{array}$ & $\begin{array}{l}\text { Prelim } 8 \\
(\mu g / g)\end{array}$ & $\begin{array}{l}\text { Average } \\
(\mu \mathrm{g} / \mathrm{g})\end{array}$ & $\begin{array}{l}\text { Uncertainty } \\
(\mu \mathrm{g} / \mathrm{g})\end{array}$ \\
\hline $\mathrm{Ag}$ & $<2.57 \mathrm{E}+02$ & $<2.51 \mathrm{E}+02$ & $<2.18 \mathrm{E}+02$ & $<2.57 \mathrm{E}+02$ & $<2.34 \mathrm{E}+02$ & $<2.40 \mathrm{E}+02$ & - \\
\hline $\mathrm{Al}$ & $8.20 \mathrm{E}+02$ & $4.52 E+03$ & $4.89 \mathrm{E}+03$ & $4.93 E+03$ & $4.75 E+03$ & $4.77 \mathrm{E}+03$ & $1.85 \mathrm{E}+02$ \\
\hline As & $<5.50 \mathrm{E}+00$ & $<3.84 \mathrm{E}+00$ & $<4.25 \mathrm{E}+00$ & $<4.94 \mathrm{E}+00$ & $<5.00 \mathrm{E}+00$ & $<4.51 \mathrm{E}+00$ & - \\
\hline B & $<3.00 \mathrm{E}+02$ & $<2.93 \mathrm{E}+02$ & $<2.54 \mathrm{E}+02$ & $<3.00 \mathrm{E}+02$ & $<2.73 \mathrm{E}+02$ & $<2.80 \mathrm{E}+02$ & - \\
\hline $\mathrm{Ba}$ & $<9.44 \mathrm{E}+01$ & $2.18 \mathrm{E}+03$ & $2.27 \mathrm{E}+03$ & $2.19 \mathrm{E}+03$ & $2.21 \mathrm{E}+03$ & $2.21 \mathrm{E}+03$ & $4.03 \mathrm{E}+01$ \\
\hline $\mathrm{Be}$ & $<5.60 \mathrm{E}+01$ & $<5.46 \mathrm{E}+01$ & $<4.73 \mathrm{E}+01$ & $<5.60 \mathrm{E}+01$ & $<5.10 \mathrm{E}+01$ & $<5.22 \mathrm{E}+01$ & - \\
\hline $\mathrm{Ca}$ & $1.90 \mathrm{E}+03$ & $2.70 \mathrm{E}+03$ & $2.65 \mathrm{E}+03$ & $2.58 \mathrm{E}+03$ & $3.14 \mathrm{E}+03$ & $2.77 \mathrm{E}+03$ & $2.53 \mathrm{E}+02$ \\
\hline Cd & $<1.22 \mathrm{E}+02$ & $<1.20 \mathrm{E}+02$ & $<1.04 \mathrm{E}+02$ & $<1.22 \mathrm{E}+02$ & $<1.12 \mathrm{E}+02$ & $<1.15 \mathrm{E}+02$ & - \\
\hline $\mathrm{Ce}$ & $<5.28 \mathrm{E}+02$ & $4.44 \mathrm{E}+03$ & $4.33 \mathrm{E}+03$ & $4.45 \mathrm{E}+03$ & $4.02 \mathrm{E}+03$ & $4.31 \mathrm{E}+03$ & $2.01 \mathrm{E}+02$ \\
\hline Co & $<6.80 \mathrm{E}+01$ & $2.48 \mathrm{E}+02$ & $2.39 \mathrm{E}+02$ & $2.45 \mathrm{E}+02$ & $2.64 \mathrm{E}+02$ & $2.49 \mathrm{E}+02$ & $1.07 \mathrm{E}+01$ \\
\hline $\mathrm{Cr}$ & $<8.40 \mathrm{E}+01$ & $3.63 \mathrm{E}+02$ & $4.14 \mathrm{E}+02$ & $3.62 \mathrm{E}+02$ & $3.76 \mathrm{E}+02$ & $3.79 E+02$ & $2.43 \mathrm{E}+01$ \\
\hline $\mathrm{Cu}$ & $<1.14 \mathrm{E}+02$ & $7.71 \mathrm{E}+02$ & $7.78 \mathrm{E}+02$ & $8.00 \mathrm{E}+02$ & $8.17 \mathrm{E}+02$ & $7.92 \mathrm{E}+02$ & $2.10 \mathrm{E}+01$ \\
\hline $\mathrm{Fe}$ & $1.71 \mathrm{E}+02$ & $4.70 \mathrm{E}+05$ & $4.51 \mathrm{E}+05$ & $4.74 \mathrm{E}+05$ & $4.53 E+05$ & $4.62 E+05$ & $1.17 \mathrm{E}+04$ \\
\hline Gd & $<3.40 \mathrm{E}+02$ & $<3.30 \mathrm{E}+02$ & $<2.86 \mathrm{E}+02$ & $<3.40 \mathrm{E}+02$ & $<3.08 \mathrm{E}+02$ & $<3.16 \mathrm{E}+02$ & - \\
\hline $\mathrm{Hg}$ & $<2.20 \mathrm{E}+01$ & $2.09 \mathrm{E}+03$ & $2.04 \mathrm{E}+03$ & $2.01 \mathrm{E}+03$ & $2.06 \mathrm{E}+03$ & $2.05 \mathrm{E}+03$ & $3.37 \mathrm{E}+01$ \\
\hline K & $<2.79 \mathrm{E}+03$ & $<2.72 \mathrm{E}+03$ & $<2.36 \mathrm{E}+03$ & $<2.79 \mathrm{E}+03$ & $<2.54 \mathrm{E}+03$ & $<2.60 \mathrm{E}+03$ & - \\
\hline $\mathrm{La}$ & $<8.64 \mathrm{E}+01$ & $1.82 \mathrm{E}+03$ & $1.79 \mathrm{E}+03$ & $1.78 \mathrm{E}+03$ & $1.75 \mathrm{E}+03$ & $1.79 \mathrm{E}+03$ & $2.89 \mathrm{E}+01$ \\
\hline $\mathrm{Li}$ & $<1.38 \mathrm{E}+02$ & $5.80 \mathrm{E}+02$ & $5.66 \mathrm{E}+02$ & $5.65 \mathrm{E}+02$ & $5.88 \mathrm{E}+02$ & $5.75 \mathrm{E}+02$ & $1.12 \mathrm{E}+01$ \\
\hline $\mathrm{Mg}$ & $<1.76 \mathrm{E}+01$ & $5.17 \mathrm{E}+02$ & $4.68 \mathrm{E}+02$ & $4.48 \mathrm{E}+02$ & $4.54 \mathrm{E}+02$ & $4.72 \mathrm{E}+02$ & $3.13 \mathrm{E}+01$ \\
\hline $\mathrm{Mn}$ & $<1.60 \mathrm{E}+01$ & $3.52 \mathrm{E}+04$ & $4.07 E+04$ & $3.60 \mathrm{E}+04$ & $3.60 \mathrm{E}+04$ & $3.70 \mathrm{E}+04$ & $2.51 \mathrm{E}+03$ \\
\hline Mo & $<5.01 \mathrm{E}+02$ & $<4.88 \mathrm{E}+02$ & $<4.23 \mathrm{E}+02$ & $<5.01 \mathrm{E}+02$ & $<4.56 \mathrm{E}+02$ & $<4.67 \mathrm{E}+02$ & - \\
\hline $\mathrm{Ni}$ & $<1.88 \mathrm{E}+02$ & $6.91 \mathrm{E}+04$ & $6.82 E+04$ & $6.93 \mathrm{E}+04$ & $6.90 \mathrm{E}+04$ & $6.89 E+04$ & $4.83 E+02$ \\
\hline $\mathrm{P}$ & $<1.35 \mathrm{E}+03$ & $<1.32 \mathrm{E}+03$ & $<1.14 \mathrm{E}+03$ & $<1.35 \mathrm{E}+03$ & $<1.23 \mathrm{E}+03$ & $<1.26 \mathrm{E}+03$ & - \\
\hline $\mathrm{Pb}$ & $<5.85 \mathrm{E}+02$ & $1.08 \mathrm{E}+03$ & $9.77 \mathrm{E}+02$ & $8.90 \mathrm{E}+02$ & $9.47 \mathrm{E}+02$ & $9.74 \mathrm{E}+02$ & $7.96 \mathrm{E}+01$ \\
\hline S & $<6.00 \mathrm{E}+03$ & $<5.85 \mathrm{E}+03$ & $<5.07 \mathrm{E}+03$ & $<6.00 \mathrm{E}+03$ & $<5.46 \mathrm{E}+03$ & $<5.60 \mathrm{E}+03$ & - \\
\hline $\mathrm{Sb}$ & $<5.50 \mathrm{E}+02$ & $<5.37 \mathrm{E}+02$ & $<4.65 \mathrm{E}+02$ & $<5.50 \mathrm{E}+02$ & $<5.01 \mathrm{E}+02$ & $<5.13 \mathrm{E}+02$ & - \\
\hline $\mathrm{Se}$ & $<1.10 \mathrm{E}+01$ & $<7.68 \mathrm{E}+00$ & $<8.50 \mathrm{E}+00$ & $<9.87 \mathrm{E}+00$ & $<1.00 \mathrm{E}+01$ & $<9.01 \mathrm{E}+00$ & - \\
\hline $\mathrm{Si}$ & $<1.85 \mathrm{E}+02$ & $8.45 \mathrm{E}+03$ & $8.93 \mathrm{E}+03$ & $8.52 \mathrm{E}+03$ & $9.58 \mathrm{E}+03$ & $8.87 \mathrm{E}+03$ & $5.19 \mathrm{E}+02$ \\
\hline Sn & $<5.15 \mathrm{E}+02$ & $<5.03 \mathrm{E}+02$ & $<4.35 \mathrm{E}+02$ & $<5.15 \mathrm{E}+02$ & $<4.68 \mathrm{E}+02$ & $<4.80 \mathrm{E}+02$ & - \\
\hline $\mathrm{Sr}$ & $<2.00 \mathrm{E}+01$ & $3.12 \mathrm{E}+02$ & $3.46 \mathrm{E}+02$ & $3.13 \mathrm{E}+02$ & $3.08 \mathrm{E}+02$ & $3.20 \mathrm{E}+02$ & $1.76 \mathrm{E}+01$ \\
\hline $\mathrm{Ti}$ & $<1.36 \mathrm{E}+01$ & $2.74 \mathrm{E}+02$ & $2.75 \mathrm{E}+02$ & $2.81 \mathrm{E}+02$ & $2.84 \mathrm{E}+02$ & $2.79 \mathrm{E}+02$ & $4.80 \mathrm{E}+00$ \\
\hline $\mathrm{U}$ & $<6.95 \mathrm{E}+03$ & $1.15 E+04$ & $1.21 \mathrm{E}+04$ & $1.02 \mathrm{E}+04$ & $1.09 \mathrm{E}+04$ & $1.12 \mathrm{E}+04$ & $8.14 \mathrm{E}+02$ \\
\hline V & $<8.32 \mathrm{E}+01$ & $<8.12 \mathrm{E}+01$ & $<7.04 \mathrm{E}+01$ & $<8.32 \mathrm{E}+01$ & $<7.58 \mathrm{E}+01$ & $<7.77 \mathrm{E}+01$ & - \\
\hline Zn & $<1.21 \mathrm{E}+02$ & $1.37 \mathrm{E}+03$ & $1.30 \mathrm{E}+03$ & $1.32 \mathrm{E}+03$ & $1.40 \mathrm{E}+03$ & $1.35 \mathrm{E}+03$ & $4.57 \mathrm{E}+01$ \\
\hline
\end{tabular}

Table 5 and Tank 6 show ICP-MS results $\left({ }^{235} \mathrm{U},{ }^{237} \mathrm{~Np},{ }^{238} \mathrm{U}\right.$, and $\left.{ }^{239} \mathrm{Pu}\right)$ for sample 1 and sample 2 , respectively. The samples were prepared by performing a peroxide fusion digestion.

\footnotetext{
${ }^{\mathrm{f}}$ Arsenic, mercury, and selenium obtained from LIMS number 300271096.

g Arsenic, mercury, and selenium obtained from LIMS number 300271092.

${ }^{\mathrm{h}}$ Arsenic, mercury, and selenium obtained from LIMS number 300271093.

${ }^{\mathrm{i}}$ Arsenic, mercury, and selenium obtained from LIMS number 300271094.

${ }^{\mathrm{j}}$ Arsenic, mercury, and selenium obtained from LIMS number 300271095.
} 
SRNL-STI-2011-00167, REV. 0

Table 5. Sample 1 ICP-MS Analysis

$\begin{array}{lllllllr} & \begin{array}{l}300271087 \\ \text { Blank }\end{array} & \begin{array}{l}300271079 \\ \text { Prelim } 1\end{array} & \begin{array}{l}300271080 \\ \text { Prelim } 2\end{array} & \begin{array}{l}300271081 \\ \text { Prelim } 3 \\ (\mu \mathrm{g} / \mathrm{g})\end{array} & \begin{array}{l}300271082 \\ \text { Prelim } 4 \\ (\mu \mathrm{g} / \mathrm{g})\end{array} & \begin{array}{l}\text { Average } \\ (\mu \mathrm{g} / \mathrm{g})\end{array} & \begin{array}{l}\text { Uncertainty } \\ (\mu \mathrm{g} / \mathrm{g})\end{array} \\ \begin{array}{l}\text { Analyte } \\ { }^{235} \mathrm{U}\end{array} & <8.00 \mathrm{E}+00 & 5.43 \mathrm{E}+01 & 5.37 \mathrm{E}+01 & 6.66 \mathrm{E}+01 & 7.50 \mathrm{E}+01 & 6.24 \mathrm{E}+01 & 1.03 \mathrm{E}+01 \\ { }^{237} \mathrm{~Np} & <4.00 \mathrm{E}+00 & 1.65 \mathrm{E}+01 & 2.76 \mathrm{E}+01 & 2.18 \mathrm{E}+01 & 1.70 \mathrm{E}+01 & 2.07 \mathrm{E}+01 & 5.17 \mathrm{E}+00 \\ { }^{238} \mathrm{U} & <1.00 \mathrm{E}+01 & 8.29 \mathrm{E}+03 & 8.62 \mathrm{E}+03 & 1.01 \mathrm{E}+04 & 1.04 \mathrm{E}+04 & 9.35 \mathrm{E}+03 & 1.05 \mathrm{E}+03 \\ { }^{239} \mathrm{Pu} & <4.00 \mathrm{E}+00 & 9.66 \mathrm{E}+01 & 1.06 \mathrm{E}+02 & 1.25 \mathrm{E}+02 & 1.23 \mathrm{E}+02 & 1.13 \mathrm{E}+02 & 1.37 \mathrm{E}+01 \\ & \text { dpm/g } & \text { dpm/g } & \text { dpm/g } & \text { dpm/g } & \text { dpm/g } & \text { dpm/g } & \\ { }^{235} \mathrm{U} & <3.84 \mathrm{E}+01 & 2.60 \mathrm{E}+02 & 2.58 \mathrm{E}+02 & 3.20 \mathrm{E}+02 & 3.60 \mathrm{E}+02 & 2.99 \mathrm{E}+02 & 4.94 \mathrm{E}+01 \\ { }^{237} \mathrm{~Np} & <6.26 \mathrm{E}+03 & 2.58 \mathrm{E}+04 & 4.32 \mathrm{E}+04 & 3.41 \mathrm{E}+04 & 2.66 \mathrm{E}+04 & 3.24 \mathrm{E}+04 & 8.09 \mathrm{E}+03 \\ { }^{238} \mathrm{U} & <7.46 \mathrm{E}+00 & 6.19 \mathrm{E}+03 & 6.43 \mathrm{E}+03 & 7.54 \mathrm{E}+03 & 7.76 \mathrm{E}+03 & 6.98 \mathrm{E}+03 & 7.85 \mathrm{E}+02 \\ { }^{239} \mathrm{Pu} & <5.52 \mathrm{E}+05 & 1.33 \mathrm{E}+07 & 1.46 \mathrm{E}+07 & 1.72 \mathrm{E}+07 & 1.70 \mathrm{E}+07 & 1.55 \mathrm{E}+07 & 1.89 \mathrm{E}+06\end{array}$

Table 6. Sample 2 ICP-MS Analysis

\begin{tabular}{|c|c|c|c|c|c|c|c|}
\hline Analyte & $\begin{array}{l}300271087 \\
\text { Blank } \\
(\mu \mathrm{g} / \mathrm{g})\end{array}$ & $\begin{array}{l}300271083 \\
\text { Prelim } 5 \\
(\mu \mathrm{g} / \mathrm{g})\end{array}$ & $\begin{array}{l}300271084 \\
\text { Prelim } 6 \\
(\mu \mathrm{g} / \mathrm{g})\end{array}$ & $\begin{array}{l}300271085 \\
\text { Prelim } 7 \\
(\mu \mathrm{g} / \mathrm{g})\end{array}$ & $\begin{array}{l}300271086 \\
\text { Prelim } 8 \\
(\mu \mathrm{g} / \mathrm{g})\end{array}$ & $\begin{array}{l}\text { Average } \\
(\mu \mathrm{g} / \mathrm{g})\end{array}$ & $\begin{array}{l}\text { Uncertainty } \\
(\mu \mathrm{g} / \mathrm{g})\end{array}$ \\
\hline${ }^{235} \mathrm{U}$ & $<8.00 \mathrm{E}+00$ & $6.48 \mathrm{E}+01$ & $6.75 E+01$ & $6.14 \mathrm{E}+01$ & $6.17 \mathrm{E}+01$ & $6.39 \mathrm{E}+01$ & $2.88 \mathrm{E}+00$ \\
\hline${ }^{237} \mathrm{~Np}$ & $<4.00 \mathrm{E}+00$ & $2.69 \mathrm{E}+01$ & $2.78 \mathrm{E}+01$ & $2.55 \mathrm{E}+01$ & $2.83 \mathrm{E}+01$ & $2.71 \mathrm{E}+01$ & $1.23 \mathrm{E}+00$ \\
\hline${ }^{238} \mathrm{U}$ & $<1.00 \mathrm{E}+01$ & $9.95 \mathrm{E}+03$ & $1.04 \mathrm{E}+04$ & $9.00 \mathrm{E}+03$ & $9.78 E+03$ & $9.78 E+03$ & $5.84 \mathrm{E}+02$ \\
\hline${ }^{239} \mathrm{Pu}$ & $\begin{array}{l}<4.00 \mathrm{E}+00 \\
\mathrm{dpm} / \mathrm{g}\end{array}$ & $\begin{array}{l}1.26 \mathrm{E}+02 \\
\mathrm{dpm} / \mathrm{g}\end{array}$ & $\begin{array}{l}1.26 \mathrm{E}+02 \\
\mathrm{dpm} / \mathrm{g}\end{array}$ & $\begin{array}{l}1.20 \mathrm{E}+02 \\
\mathrm{dpm} / \mathrm{g}\end{array}$ & $\begin{array}{l}1.29 \mathrm{E}+02 \\
\mathrm{dpm} / \mathrm{g}\end{array}$ & $\begin{array}{l}1.25 \mathrm{E}+02 \\
\mathrm{dpm} / \mathrm{g}\end{array}$ & $3.77 \mathrm{E}+00$ \\
\hline${ }^{235} \mathrm{U}$ & $<3.84 \mathrm{E}+01$ & $3.11 \mathrm{E}+02$ & $3.24 \mathrm{E}+02$ & $2.95 E+02$ & $2.96 \mathrm{E}+02$ & $3.06 \mathrm{E}+02$ & $1.38 E+01$ \\
\hline${ }^{237} \mathrm{~Np}$ & $<6.26 \mathrm{E}+03$ & $4.21 \mathrm{E}+04$ & $4.35 \mathrm{E}+04$ & $3.99 \mathrm{E}+04$ & $4.43 \mathrm{E}+04$ & $4.24 \mathrm{E}+04$ & $1.92 \mathrm{E}+03$ \\
\hline${ }^{238} \mathrm{U}$ & $<7.46 \mathrm{E}+00$ & $7.43 \mathrm{E}+03$ & $7.76 \mathrm{E}+03$ & $6.72 \mathrm{E}+03$ & $7.30 \mathrm{E}+03$ & $7.30 \mathrm{E}+03$ & $4.36 \mathrm{E}+02$ \\
\hline${ }^{239} \mathrm{Pu}$ & $<5.52 \mathrm{E}+05$ & $1.74 \mathrm{E}+07$ & $1.74 \mathrm{E}+07$ & $1.66 \mathrm{E}+07$ & $1.78 \mathrm{E}+07$ & $1.73 E+07$ & $5.21 \mathrm{E}+05$ \\
\hline
\end{tabular}

Table 7 and Table 8 show the radionuclide analysis of sample 1 and sample 2, respectively.

Table 9 compares the concentrations of select cations and radionuclides in the Feed and Bleed Residual solids, the Process Sample ${ }^{3}$, and a sample collected prior to Mechanical Sludge Removal in Tank $5 F^{4}$. Most of the species show good agreement between their concentration in the Feed and Bleed Sample and their concentration in the Process Sample.

The following species showed lower concentrations in the Feed and Bleed Samples than in the Process Sample. The chromium concentration was 75\% lower in the Feed and Bleed samples than in the process sample. The Nickel concentration was 22\% lower in the Feed and Bleed Sample than in the Process Sample. The silicon concentration was about 50\% lower in the Feed and Bleed Sample than in the Process Sample.

The following samples showed higher concentrations in the Feed and Bleed Samples than in the Process Sample. The aluminum concentration was approximately four times larger in the Feed and Bleed Sample. The barium concentration was approximately two times larger in the Feed and Bleed Sample. The calcium concentration was approximately nine times larger in the Feed and Bleed Sample. The calcium concentration in the blank is the same order of magnitude as the samples. Therefore, the calcium in the samples might be from the dissolution reagents. The strontium (total strontium and ${ }^{90} \mathrm{Sr}$ ) concentration was approximately three times larger in the Feed and Bleed Sample. The uranium concentration was more than six times larger in the Feed 
and Bleed Sample. The ${ }^{137}$ Cs was approximately nine times larger in the Feed and Bleed Sample. We are not sure of the reason for these differences.

Figures 1 and 2 show the particle size distribution of four samples analyzed by Microtrac. The samples had a median particle size of $60-109$ micron, a $90^{\text {th }}$ percentile particle size of $350-$ 640 micron, and a maximum particle size of 704 micron or larger. Figure 2 shows peaks between 296 and 704 micron. Some of the samples show two multiple peaks. Figure 2 shows a flat distribution between 0.5 micron and 100 micron.

Figures 3 and 4 show the particle size distribution of four samples by SEM. The samples had a median particle size of $600-700$ micron, and an $85^{\text {th }}$ percentile of 900 micron. Figure 4 shows a peak at 900 micron. More than $90 \%$ of the particles are greater than 200 micron.

Figures 5 and 6 compare the particle size measures by the Microtrac and SEM methods. The particle size distribution of the particles measured by Microtrac is smaller than the particle size distribution of the particles measured by SEM. One likely cause of this difference is the shearing of the particles that occurs when their size is measured by Microtrac.

Table 7. Sample 1 Radionuclide Analysis

\begin{tabular}{|c|c|c|c|c|c|c|c|}
\hline Analyte & $\begin{array}{l}300271087 \\
\text { Blank } \\
(\mathrm{dpm} / \mathrm{g})\end{array}$ & $\begin{array}{l}300271079 \\
\text { Prelim } 1 \\
(\mathrm{dpm} / \mathrm{g})\end{array}$ & $\begin{array}{l}300271080 \\
\text { Prelim } 2 \\
(\mathrm{dpm} / \mathrm{g})\end{array}$ & $\begin{array}{l}300271081 \\
\text { Prelim } 3 \\
\text { (dpm/g) }\end{array}$ & $\begin{array}{l}300271082 \\
\text { Prelim } 4 \\
\text { (dpm/g) }\end{array}$ & $\begin{array}{l}\text { Average } \\
\text { (dpm/g) }\end{array}$ & $\begin{array}{l}\text { Uncertainty } \\
(\mathrm{dpm} / \mathrm{g})\end{array}$ \\
\hline${ }^{60} \mathrm{Co}$ & $<3.42 \mathrm{E}+05$ & $1.69 \mathrm{E}+07$ & $1.67 \mathrm{E}+07$ & $1.91 \mathrm{E}+07$ & $1.87 \mathrm{E}+07$ & $1.79 \mathrm{E}+07$ & $1.23 \mathrm{E}+06$ \\
\hline${ }^{90} \mathrm{Sr}$ & $<8.78 \mathrm{E}+07$ & $1.96 \mathrm{E}+10$ & $2.33 \mathrm{E}+10$ & $3.62 \mathrm{E}+10$ & $4.19 \mathrm{E}+10$ & $3.03 E+10$ & $1.05 \mathrm{E}+10$ \\
\hline${ }^{99} \mathrm{Tc}^{\mathrm{k}}$ & $<5.99 \mathrm{E}+01$ & $3.82 E+03$ & $2.25 E+03$ & $3.19 E+03$ & $3.86 \mathrm{E}+03$ & $3.28 \mathrm{E}+03$ & $7.52 \mathrm{E}+02$ \\
\hline${ }^{37} \mathrm{Cs}$ & $<3.46 \mathrm{E}+05$ & $6.08 \mathrm{E}+08$ & $6.56 \mathrm{E}+08$ & $7.19 \mathrm{E}+08$ & $7.29 E+08$ & $6.78 \mathrm{E}+08$ & $5.68 \mathrm{E}+07$ \\
\hline${ }^{154} \mathrm{Eu}$ & $<3.80 \mathrm{E}+05$ & $6.39 \mathrm{E}+07$ & $6.60 \mathrm{E}+07$ & $7.67 \mathrm{E}+07$ & $7.26 \mathrm{E}+07$ & $6.98 \mathrm{E}+07$ & $5.91 E+06$ \\
\hline${ }^{155} \mathrm{Eu}$ & & & & $1.18 \mathrm{E}+07$ & & $\mathrm{n} / \mathrm{a}$ & \\
\hline${ }^{233} \mathrm{U}$ & $<3.09 \mathrm{E}+03$ & $<2.29 \mathrm{E}+02$ & $<2.24 \mathrm{E}+02$ & $<2.55 \mathrm{E}+02$ & $<3.20 \mathrm{E}+02$ & $<2.72 \mathrm{E}+02$ & - \\
\hline${ }^{234} \mathrm{U}$ & $<1.98 \mathrm{E}+03$ & $6.53 E+03$ & $6.66 \mathrm{E}+03$ & $7.70 \mathrm{E}+03$ & $9.24 \mathrm{E}+03$ & $7.53 \mathrm{E}+03$ & $1.25 \mathrm{E}+03$ \\
\hline${ }^{235} \mathrm{U}$ & $<6.90 \mathrm{E}-01$ & $2.60 \mathrm{E}+02$ & $2.73 E+02$ & $3.17 E+02$ & $3.263 \mathrm{E}+02$ & $2.94 \mathrm{E}+02$ & $3.27 \mathrm{E}+01$ \\
\hline${ }^{36} \mathrm{U}$ & $<2.07 \mathrm{E}+01$ & $3.13 \mathrm{E}+02$ & $3.20 \mathrm{E}+02$ & $3.73 \mathrm{E}+02$ & $4.44 \mathrm{E}+02$ & $3.62 \mathrm{E}+02$ & $6.06 \mathrm{E}+01$ \\
\hline${ }^{38} \mathrm{U}$ & $<7.46 \mathrm{E}+00$ & $6.19 E+03$ & $6.44 \mathrm{E}+03$ & $7.55 \mathrm{E}+03$ & $7.77 E+03$ & $6.99 \mathrm{E}+03$ & $7.87 \mathrm{E}+02$ \\
\hline${ }^{241} \mathrm{Pu}$ & $<4.08 \mathrm{E}+06$ & $<3.57 \mathrm{E}+07$ & $<1.79 \mathrm{E}+07$ & $<2.02 \mathrm{E}+07$ & $<2.86 \mathrm{E}+07$ & $<2.56 \mathrm{E}+07$ & - \\
\hline${ }^{238} \mathrm{Pu}$ & $<1.73 \mathrm{E}+06$ & $5.21 \mathrm{E}+06$ & $4.37 \mathrm{E}+06$ & $3.33 E+06$ & $5.20 \mathrm{E}+06$ & $4.53 \mathrm{E}+06$ & $8.90 \mathrm{E}+05$ \\
\hline${ }^{239 / 240} \mathrm{Pu}$ & $3.40 \mathrm{E}+06$ & $1.29 \mathrm{E}+07$ & $1.73 \mathrm{E}+07$ & $1.58 \mathrm{E}+07$ & $2.37 \mathrm{E}+07$ & $1.74 \mathrm{E}+07$ & $4.56 \mathrm{E}+06$ \\
\hline${ }^{239} \mathrm{Pu}$ & $<7.30 \mathrm{E}+05$ & $1.03 \mathrm{E}+07$ & $1.37 \mathrm{E}+07$ & $1.26 \mathrm{E}+07$ & $1.87 \mathrm{E}+07$ & $1.38 \mathrm{E}+07$ & $3.57 \mathrm{E}+06$ \\
\hline${ }^{240} \mathrm{Pu}$ & $<2.66 \mathrm{E}+06$ & $2.60 \mathrm{E}+06$ & $3.53 \mathrm{E}+06$ & $3.24 \mathrm{E}+06$ & $4.938 \mathrm{E}+06$ & $3.57 \mathrm{E}+06$ & $9.83 \mathrm{E}+05$ \\
\hline${ }^{242} \mathrm{Pu}$ & $<4.64 \mathrm{E}+04$ & $4.95 \mathrm{E}+02$ & $6.35 \mathrm{E}+02$ & $5.42 E+02$ & $7.53 E+02$ & $6.06 \mathrm{E}+02$ & $1.14 \mathrm{E}+02$ \\
\hline${ }^{244} \mathrm{Pu}$ & $<3.24 \mathrm{E}+02$ & $<2.64 \mathrm{E}+00$ & $<4.02 \mathrm{E}+00$ & $<2.80 \mathrm{E}+00$ & $<2.33 \mathrm{E}+00$ & $<2.95 \mathrm{E}+00$ & - \\
\hline${ }^{241} \mathrm{Am}$ & $<1.60 \mathrm{E}+06$ & $1.22 \mathrm{E}+08$ & $1.34 \mathrm{E}+08$ & $1.49 \mathrm{E}+08$ & $1.35 \mathrm{E}+08$ & $1.35 \mathrm{E}+08$ & $1.10 \mathrm{E}+07$ \\
\hline${ }^{243} \mathrm{Am}$ & $<4.47 \mathrm{E}+05$ & $8.60 \mathrm{E}+05$ & $9.84 \mathrm{E}+05$ & $1.12 \mathrm{E}+06$ & $1.02 \mathrm{E}+06$ & $9.96 \mathrm{E}+05$ & $1.07 \mathrm{E}+05$ \\
\hline${ }^{242 \mathrm{~m}} \mathrm{Am}$ & $<1.61 \mathrm{E}+05$ & $3.59 \mathrm{E}+05$ & $3.68 \mathrm{E}+05$ & $3.64 \mathrm{E}+05$ & $3.43 \mathrm{E}+05$ & $3.59 \mathrm{E}+05$ & $1.10 \mathrm{E}+04$ \\
\hline${ }^{243} \mathrm{Cm}$ & $<1.66 \mathrm{E}+06$ & $<6.10 \mathrm{E}+05$ & $<2.23 \mathrm{E}+06$ & $<5.75 \mathrm{E}+05$ & $<7.85 \mathrm{E}+05$ & $<1.05 \mathrm{E}+06$ & - \\
\hline${ }^{245} \mathrm{Cm}$ & $<1.36 \mathrm{E}+06$ & $<7.40 \mathrm{E}+05$ & $<5.64 \mathrm{E}+05$ & $<4.82 \mathrm{E}+05$ & $<4.01 \mathrm{E}+05$ & $<5.47 \mathrm{E}+05$ & - \\
\hline${ }^{247} \mathrm{Cm}$ & $<2.09 \mathrm{E}+06$ & $<3.52 \mathrm{E}+05$ & $<2.25 \mathrm{E}+05$ & $<2.64 \mathrm{E}+05$ & $<1.60 \mathrm{E}+05$ & $<2.50 \mathrm{E}+05$ & - \\
\hline${ }^{249} \mathrm{Cf}$ & $<2.09 \mathrm{E}+06$ & $<3.62 \mathrm{E}+05$ & $<2.26 \mathrm{E}+05$ & $<2.60 \mathrm{E}+05$ & $<1.61 \mathrm{E}+05$ & $<2.52 \mathrm{E}+05$ & - \\
\hline${ }^{251} \mathrm{Cf}$ & $<1.54 \mathrm{E}+06$ & $<2.23 \mathrm{E}+05$ & $<1.99 \mathrm{E}+05$ & $<2.16 \mathrm{E}+05$ & $<1.38 \mathrm{E}+05$ & $<1.94 \mathrm{E}+05$ & - \\
\hline${ }^{242} \mathrm{Cm}$ & $<1.33 \mathrm{E}+05$ & $2.97 \mathrm{E}+05$ & $3.04 \mathrm{E}+05$ & $3.01 \mathrm{E}+05$ & $2.83 E+05$ & $2.96 \mathrm{E}+05$ & $9.29 E+03$ \\
\hline${ }^{244} \mathrm{Cm}$ & $<5.28 \mathrm{E}+05$ & $7.20 \mathrm{E}+06$ & $7.02 \mathrm{E}+06$ & $8.45 E+06$ & $7.54 \mathrm{E}+06$ & $7.55 \mathrm{E}+06$ & $6.36 \mathrm{E}+05$ \\
\hline
\end{tabular}

${ }^{\mathrm{k}}{ }^{99}$ Tc samples had LIMS numbers of 300271105, 300271097, 300271098, 300271099, 300271100 
SRNL-STI-2011-00167, REV. 0

Table 8. Sample 2 Radionuclide Analysis

\begin{tabular}{|c|c|c|c|c|c|c|c|}
\hline Analyte & $\begin{array}{l}300271087 \\
\text { Blank } \\
(\mathrm{dpm} / \mathrm{g})\end{array}$ & $\begin{array}{l}300271083 \\
\text { Prelim } 5 \\
(\mathrm{dpm} / \mathrm{g})\end{array}$ & $\begin{array}{l}300271084 \\
\text { Prelim } 6 \\
(\mathrm{dpm} / \mathrm{g})\end{array}$ & $\begin{array}{l}300271085 \\
\text { Prelim } 7 \\
(\mathrm{dpm} / \mathrm{g})\end{array}$ & $\begin{array}{l}300271086 \\
\text { Prelim } 8 \\
(\mathrm{dpm} / \mathrm{g})\end{array}$ & $\begin{array}{l}\text { Average } \\
\text { (dpm/g) }\end{array}$ & $\begin{array}{l}\text { Uncertainty } \\
\text { (dpm/g) }\end{array}$ \\
\hline${ }^{60} \mathrm{Co}$ & $<3.42 \mathrm{E}+05$ & $2.00 \mathrm{E}+07$ & $1.96 \mathrm{E}+07$ & $2.01 \mathrm{E}+07$ & $1.87 \mathrm{E}+07$ & $1.96 \mathrm{E}+07$ & $6.38 \mathrm{E}+05$ \\
\hline${ }^{90} \mathrm{Sr}$ & $<8.78 \mathrm{E}+07$ & $2.52 \mathrm{E}+10$ & $3.72 E+10$ & $3.26 \mathrm{E}+10$ & $2.64 \mathrm{E}+10$ & $3.04 \mathrm{E}+10$ & $5.60 \mathrm{E}+09$ \\
\hline${ }^{99} \mathrm{Tc}^{\mathrm{l}}$ & $<5.99 \mathrm{E}+01$ & $4.40 \mathrm{E}+03$ & $3.52 E+03$ & $2.92 E+03$ & $5.65 E+03$ & $4.12 \mathrm{E}+03$ & $1.19 E+03$ \\
\hline${ }^{137} \mathrm{Cs}$ & $<3.46 \mathrm{E}+05$ & $6.66 \mathrm{E}+08$ & $7.11 \mathrm{E}+08$ & $6.47 \mathrm{E}+08$ & $6.93 E+08$ & $6.79 \mathrm{E}+08$ & $2.84 \mathrm{E}+07$ \\
\hline${ }^{154} \mathrm{Eu}$ & $<3.80 \mathrm{E}+05$ & $8.95 \mathrm{E}+07$ & $8.56 \mathrm{E}+07$ & $9.03 \mathrm{E}+07$ & $8.62 E+07$ & $8.79 \mathrm{E}+07$ & $2.35 E+06$ \\
\hline${ }^{155} \mathrm{Eu}$ & \# & \# & \# & \# & & $\mathrm{n} / \mathrm{a}$ & - \\
\hline${ }^{233} \mathrm{U}$ & $<3.09 \mathrm{E}+03$ & $<2.49 \mathrm{E}+02$ & $<2.01 \mathrm{E}+02$ & $<3.17 \mathrm{E}+02$ & $<2.58 \mathrm{E}+02$ & $<2.56 \mathrm{E}+02$ & - \\
\hline${ }^{234} \mathrm{U}$ & $<1.987 \mathrm{E}+03$ & $7.02 \mathrm{E}+03$ & $8.24 \mathrm{E}+03$ & $7.04 \mathrm{E}+03$ & $7.57 \mathrm{E}+03$ & $7.46 \mathrm{E}+03$ & $5.75 \mathrm{E}+02$ \\
\hline${ }^{235} \mathrm{U}$ & $<6.90 \mathrm{E}-01$ & $3.15 \mathrm{E}+02$ & $3.24 \mathrm{E}+02$ & $2.82 E+02$ & $3.04 \mathrm{E}+02$ & $3.06 \mathrm{E}+02$ & $1.82 \mathrm{E}+01$ \\
\hline${ }^{236} \mathrm{U}$ & $<2.07 \mathrm{E}+01$ & $3.37 \mathrm{E}+02$ & $4.00 \mathrm{E}+02$ & $3.40 \mathrm{E}+02$ & $3.71 E+02$ & $3.62 E+02$ & $2.941 \mathrm{E}+01$ \\
\hline${ }^{238} \mathrm{U}$ & $<7.46 \mathrm{E}+00$ & $7.41 \mathrm{E}+03$ & $7.77 \mathrm{E}+03$ & $6.70 \mathrm{E}+03$ & $7.30 \mathrm{E}+03$ & 7.30E+03 & 4.43E+02 \\
\hline${ }^{241} \mathrm{Pu}$ & $<4.08 \mathrm{E}+06$ & $<2.92 \mathrm{E}+07$ & $<1.90 \mathrm{E}+07$ & $<2.81 \mathrm{E}+07$ & $<1.54 \mathrm{E}+07$ & $<2.29 \mathrm{E}+07$ & - \\
\hline${ }^{238} \mathrm{Pu}$ & $<1.73 \mathrm{E}+06$ & $7.51 \mathrm{E}+06$ & $4.61 \mathrm{E}+06$ & $5.73 E+06$ & $2.58 \mathrm{E}+06$ & $5.11 \mathrm{E}+06$ & $2.07 E+06$ \\
\hline${ }^{239 / 240} \mathrm{Pu}$ & $3.40 \mathrm{E}+06$ & $2.11 \mathrm{E}+07$ & $3.14 \mathrm{E}+07$ & $2.09 \mathrm{E}+07$ & $2.55 \mathrm{E}+07$ & $2.47 \mathrm{E}+07$ & $4.93 E+06$ \\
\hline${ }^{239} \mathrm{Pu}$ & $<7.30 \mathrm{E}+05$ & $1.68 \mathrm{E}+07$ & $2.51 E+07$ & $1.67 \mathrm{E}+07$ & $2.04 \mathrm{E}+07$ & $1.97 \mathrm{E}+07$ & $3.95 E+06$ \\
\hline${ }^{240} \mathrm{Pu}$ & $<2.66 \mathrm{E}+06$ & $4.28 \mathrm{E}+06$ & $6.35 E+06$ & 4.17E+06 & $5.15 E+06$ & $4.99 E+06$ & $1.01 \mathrm{E}+06$ \\
\hline${ }^{242} \mathrm{Pu}$ & $<4.64 \mathrm{E}+04$ & $7.02 \mathrm{E}+02$ & $9.99 \mathrm{E}+02$ & $6.77 \mathrm{E}+02$ & $<1.97 \mathrm{E}+03$ & $7.93 \mathrm{E}+02$ & $1.79 E+02$ \\
\hline${ }^{244} \mathrm{Pu}$ & $<3.24 \mathrm{E}+02$ & $<3.62 \mathrm{E}+00$ & $<5.79 \mathrm{E}+00$ & $<4.11 \mathrm{E}+00$ & $<1.37 \mathrm{E}+01$ & $<6.82 \mathrm{E}+00$ & - \\
\hline${ }^{241} \mathrm{Am}$ & $<1.60 \mathrm{E}+06$ & $1.87 \mathrm{E}+08$ & $1.76 \mathrm{E}+08$ & $1.67 \mathrm{E}+08$ & $1.76 \mathrm{E}+08$ & $1.77 \mathrm{E}+08$ & $8.19 \mathrm{E}+06$ \\
\hline${ }^{243} \mathrm{Am}$ & $<4.47 \mathrm{E}+05$ & $1.32 \mathrm{E}+06$ & $1.21 \mathrm{E}+06$ & $1.21 \mathrm{E}+06$ & $1.35 \mathrm{E}+06$ & $1.27 \mathrm{E}+06$ & $7.32 \mathrm{E}+04$ \\
\hline${ }^{242 m} \mathrm{Am}$ & $<1.61 \mathrm{E}+05$ & $4.48 \mathrm{E}+05$ & $4.97 \mathrm{E}+05$ & $4.83 E+05$ & $5.01 \mathrm{E}+05$ & $4.82 \mathrm{E}+05$ & $2.41 \mathrm{E}+04$ \\
\hline${ }^{243} \mathrm{Cm}$ & $<1.66 \mathrm{E}+06$ & $<1.02 \mathrm{E}+06$ & $<1.18 \mathrm{E}+06$ & $<6.81 \mathrm{E}+05$ & $<1.98 \mathrm{E}+06$ & $<1.22 \mathrm{E}+06$ & - \\
\hline${ }^{245} \mathrm{Cm}$ & $<1.36 \mathrm{E}+06$ & $<9.65 \mathrm{E}+05$ & $<4.52 \mathrm{E}+05$ & $<5.71 \mathrm{E}+05$ & $<4.39 \mathrm{E}+05$ & $<6.07 \mathrm{E}+05$ & - \\
\hline${ }^{247} \mathrm{Cm}$ & $<2.09 \mathrm{E}+06$ & $<3.51 \mathrm{E}+05$ & $<1.68 \mathrm{E}+05$ & $<3.12 \mathrm{E}+05$ & $<1.78 \mathrm{E}+05$ & $<2.52 \mathrm{E}+05$ & - \\
\hline${ }^{249} \mathrm{Cf}$ & $<2.09 \mathrm{E}+06$ & $<3.51 \mathrm{E}+05$ & $<1.69 \mathrm{E}+05$ & $<3.09 \mathrm{E}+05$ & $<1.77 \mathrm{E}+05$ & $<2.52 \mathrm{E}+05$ & - \\
\hline${ }^{251} \mathrm{Cf}$ & $<1.54 \mathrm{E}+06$ & $<2.96 \mathrm{E}+05$ & $<1.40 \mathrm{E}+05$ & $<2.55 \mathrm{E}+05$ & $<1.56 \mathrm{E}+05$ & $<2.12 \mathrm{E}+05$ & - \\
\hline${ }^{242} \mathrm{Cm}$ & $<1.33 \mathrm{E}+05$ & $3.71 \mathrm{E}+05$ & $4.11 \mathrm{E}+05$ & $3.99 \mathrm{E}+05$ & $4.14 \mathrm{E}+05$ & $3.99 \mathrm{E}+05$ & $1.96 \mathrm{E}+04$ \\
\hline${ }^{244} \mathrm{Cm}$ & $<5.28 \mathrm{E}+05$ & $1.05 \mathrm{E}+07$ & $1.15 \mathrm{E}+07$ & $1.05 \mathrm{E}+07$ & $1.15 \mathrm{E}+07$ & $1.10 \mathrm{E}+07$ & $5.77 \mathrm{E}+05$ \\
\hline
\end{tabular}

\footnotetext{
${ }^{199}$ Tc samples had LIMS numbers of 300271105, 300271101, 300271102, 300271103, 300271104
} 
Table 9. Comparison of Feed and Bleed Sample with 2007 Tank 5F Sample and Tank 5F Process Sample

Species

$\mathrm{Al}$

$\mathrm{Ba}$

$\mathrm{Ca}$

$\mathrm{Cr}$

$\mathrm{Fe}$

$\mathrm{Hg}$

$\mathrm{Mg}$

$\mathrm{Mn}$

$\mathrm{Ni}$

$\mathrm{Si}$

$\mathrm{Sr}$

U

${ }^{60} \mathrm{Co}$

${ }^{90} \mathrm{Sr}$

${ }^{99} \mathrm{Tc}$

${ }^{137} \mathrm{Cs}$

${ }^{154} \mathrm{Eu}$

${ }^{241} \mathrm{Pu}$

${ }^{238} \mathrm{Pu}$

${ }^{239 / 240} \mathrm{Pu}$

${ }^{241} \mathrm{Am}$

${ }^{243} \mathrm{Am}$

${ }^{242 \mathrm{~m}} \mathrm{Am}$

${ }^{243} \mathrm{Cm}$

${ }^{245} \mathrm{Cm}$

${ }^{247} \mathrm{Cm}$

${ }^{249} \mathrm{Cf}$

${ }^{251} \mathrm{Cf}$

${ }^{242} \mathrm{Cm}$

${ }^{244} \mathrm{Cm}$

* Not reported
Sample 1

( $\mu \mathrm{g} / \mathrm{g}$ )

5,110

2,180

2,530

354

475,000

2,070

480

38,800

60,000

8,660

365

10,200

(dpm/g)

1.79E07

3.03E10

$3.28 \mathrm{E} 03$

$6.78 \mathrm{E} 08$

$6.98 \mathrm{E} 07$

$<2.56 \mathrm{E} 07$

4.53E06

$1.74 \mathrm{E} 07$

$1.35 \mathrm{E} 08$

$9.96 \mathrm{E} 05$

3.59E05

$<1.05 \mathrm{E} 06$

$<5.47 \mathrm{E} 05$

$<2.50 \mathrm{E} 05$

$<2.52 \mathrm{E} 05$

$<1.94 \mathrm{E} 05$

$2.96 \mathrm{E} 05$

7.55E06
Sample 2

( $\mu \mathrm{g} / \mathrm{g}$ )

4,770

2,210

2,770

379

462,000

2,050

472

37,000

68,900

8,870

320

11,200

(dpm/g)

1.96E07

3.04E10

4.12E03

6.79E08

8.79E07

$<2.29 \mathrm{E} 07$

5.11E06

2.47E07

1.77E08

$1.27 \mathrm{E} 06$

4.82E05

$<1.22 \mathrm{E} 06$

$<6.07 \mathrm{E} 05$

$<2.52 \mathrm{E} 05$

$<2.52 \mathrm{E} 05$

$<2.12 \mathrm{E} 05$

3.99E05

1.10E07
Process Sample 2007 Sample

( $\mu \mathrm{g} / \mathrm{g})$

1290

1090

303

1470

177,000

1480

409

34,600

83,000

19,100

108

$<1560$

(dpm/g)

2.4E07

$1.2 \mathrm{E} 10$

$<5.2 \mathrm{E} 03$

7.7E07

$6.5 \mathrm{E} 07$

$2.9 \mathrm{E} 07$

6.3E06

2.4E07

$1.4 \mathrm{E} 08$

$3.5 \mathrm{E} 06$

$4.0 \mathrm{E} 05$

$<1.1 \mathrm{E} 06$

$<1.0 \mathrm{E} 06$

$<2.6 \mathrm{E} 05$

$<2.7 \mathrm{E} 05$

$<4.2 \mathrm{E} 05$

3.5E05

1.1E07
( $\mu \mathrm{g} / \mathrm{g})$

14,400

1,820

3,470

$<1,100$

373,000

1,290

$<850$

68,400

44,500

11,800

1,500

100,000

(dpm/g)

3.1E07

8.1E10

2.9E04

2.3E09

1.8E07

4.3E06

$1.9 \mathrm{E} 07$

$1.1 \mathrm{E} 08$

7.4E05

3.2E05

2.7E05

8.5E06 


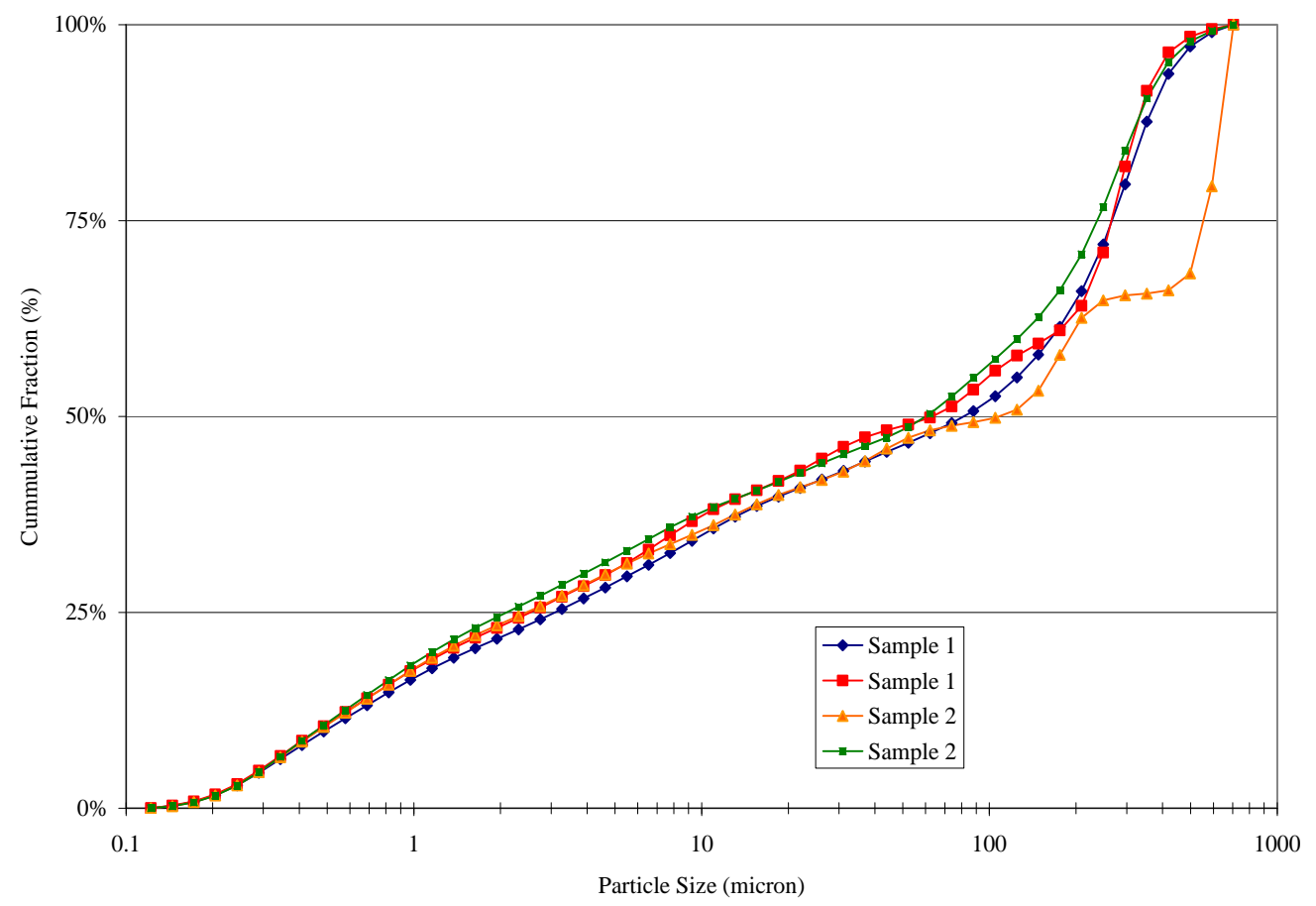

Figure 1. Particle Size Distribution of Tank 5F Feed and Bleed Residual Solids Samples by Microtrac

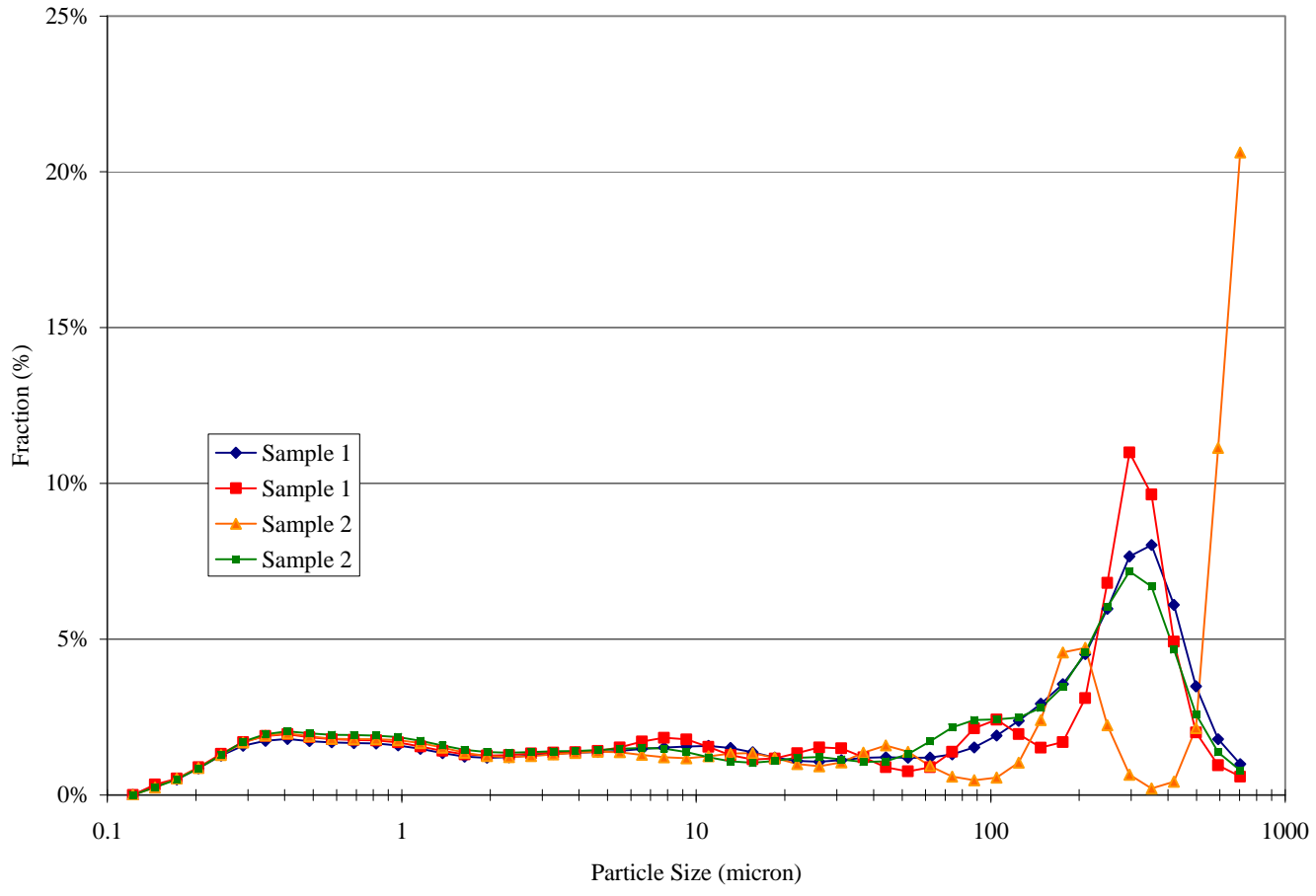

Figure 2. Particle Size Distribution of Tank 5F Feed and Bleed Residual Solids Samples by Microtrac 
Cum Fract

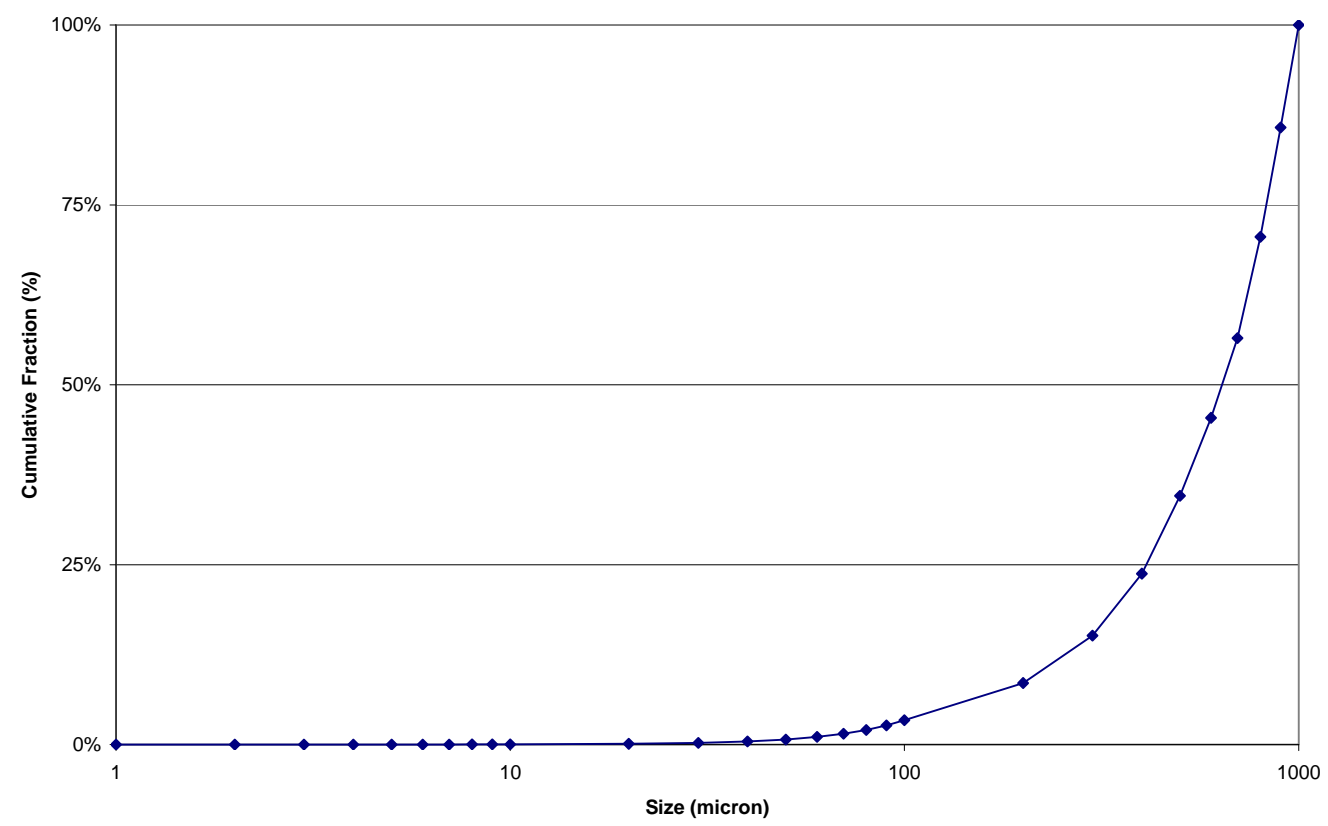

Figure 3. Particle Size Distribution of Tank 5F Feed and Bleed Residual Solids Samples by SEM

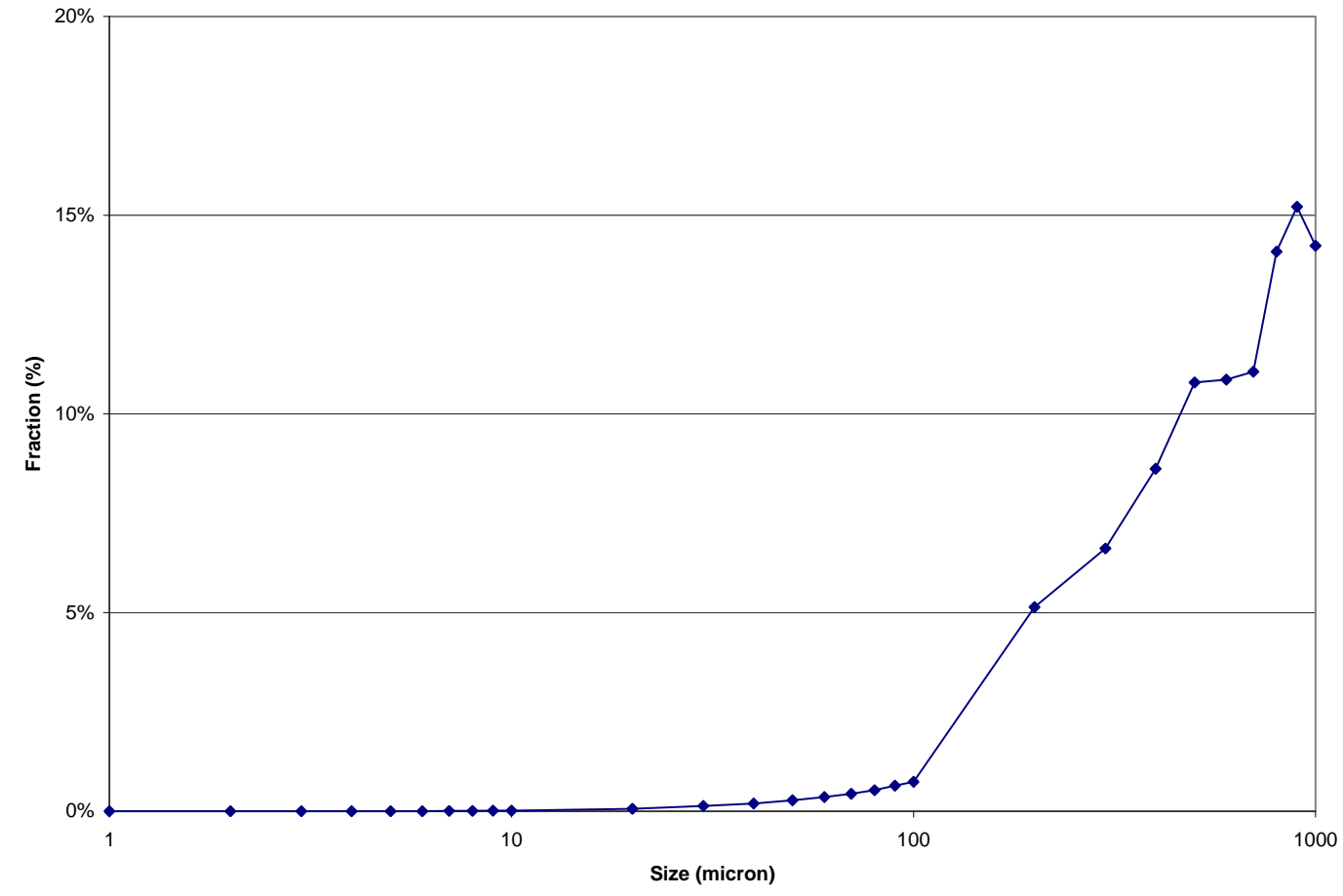

Figure 4. Particle Size Distribution of Tank 5F Feed and Bleed Residual Solids Samples by SEM 


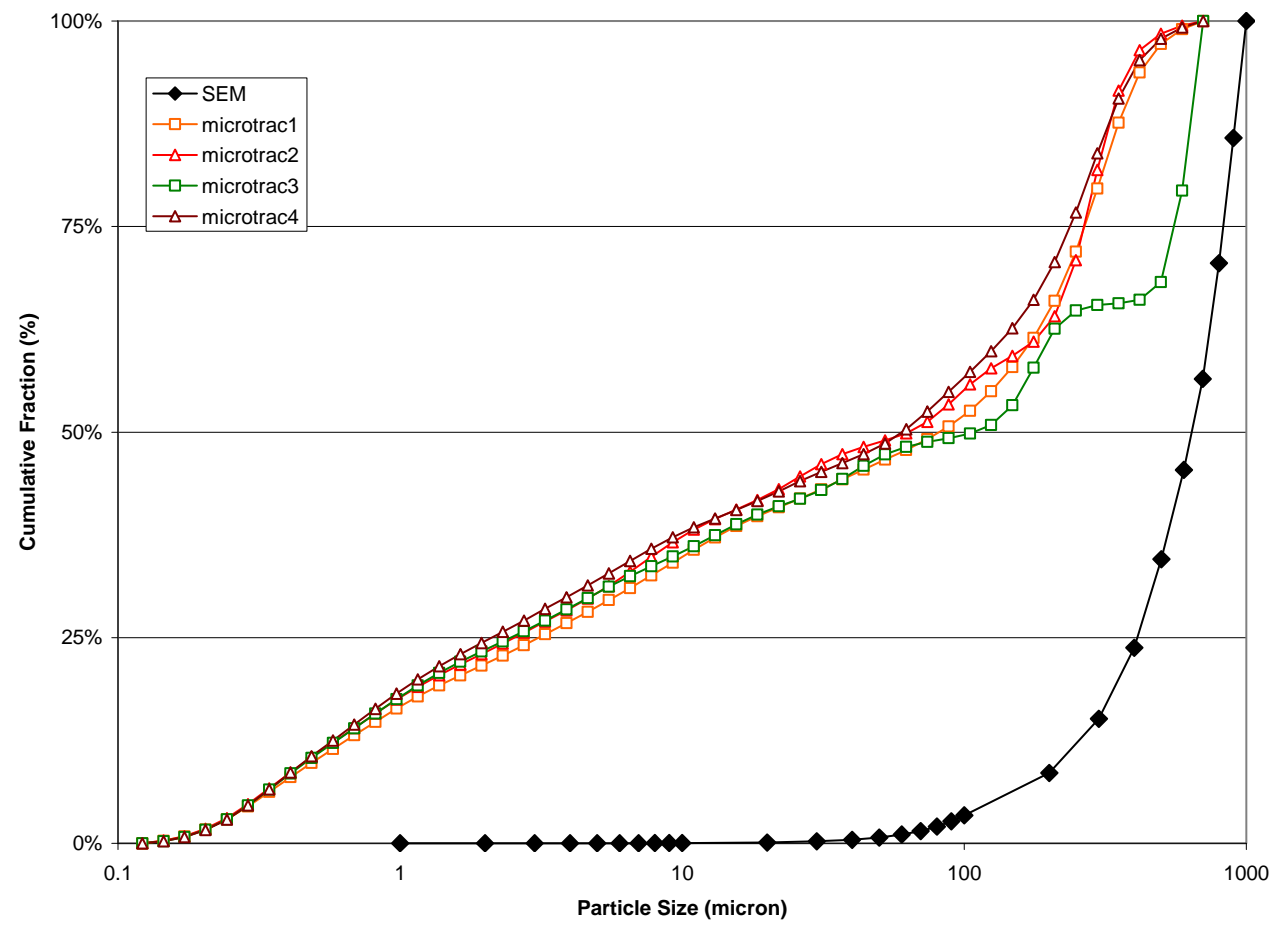

Figure 5. Comparison of Particle Size Measurements by Microtrac and SEM

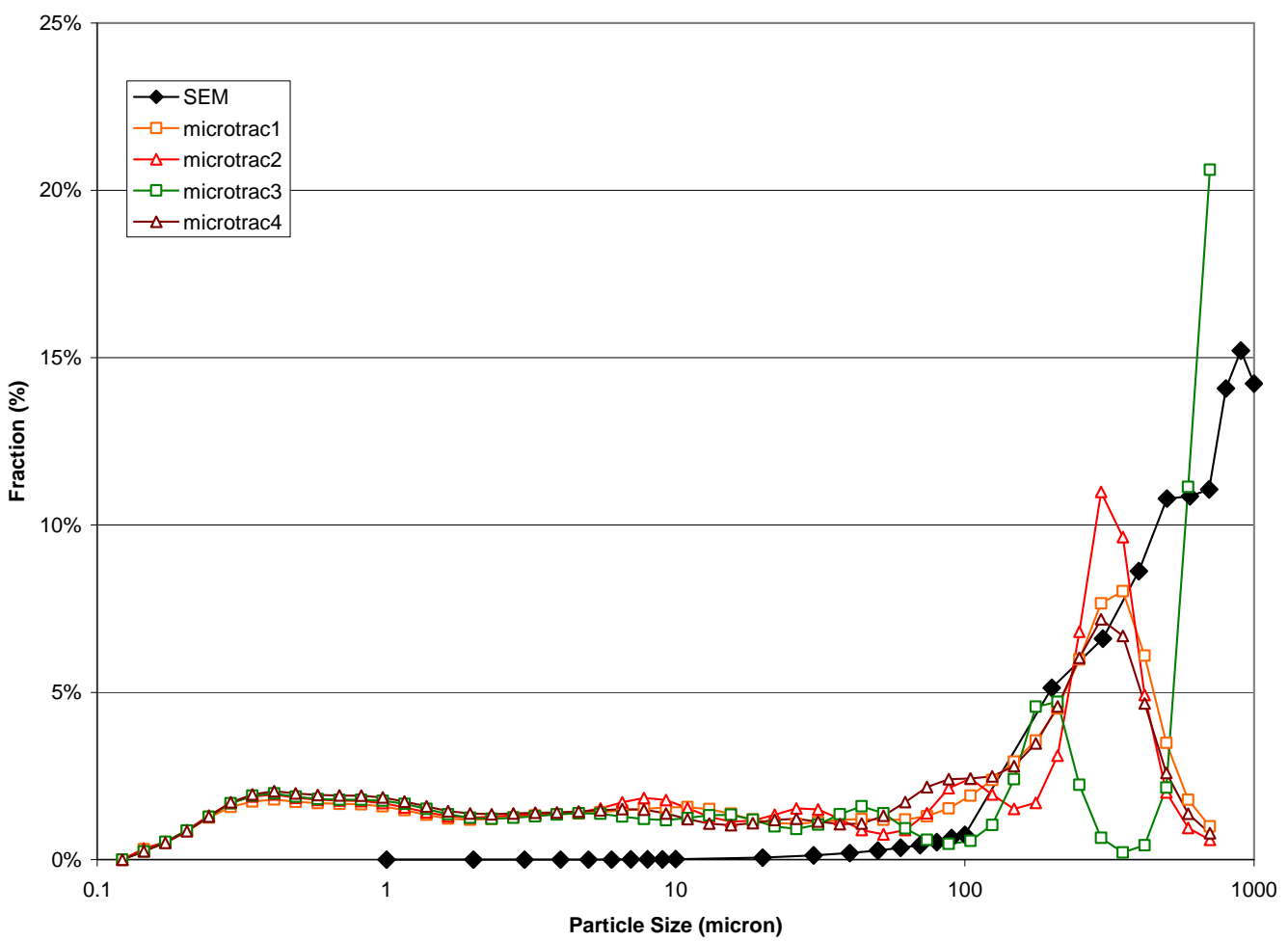

Figure 6. Comparison of Particle Size Measurements by Microtrac and SEM 


\subsection{CONCLUSIONS}

The conclusions from this analysis follow.

- All anions measured had a concentration less than $250 \mathrm{mg} / \mathrm{kg}$, except for oxalate, which had a concentration of $2100-2400 \mathrm{mg} / \mathrm{kg}$.

- The measured cations with the highest concentration were iron (432,000 - 519,000 $\mathrm{mg} / \mathrm{kg}$ ), nickel (54,600 - 69,300 mg/kg), and manganese (35,200 - 42,100 mg/kg). All other cations measured less than $13,000 \mathrm{mg} / \mathrm{kg}$.

- The radionuclides present in the highest concentration are ${ }^{90} \mathrm{Sr}\left(3.0 \times 10^{10} \mathrm{dpm} / \mathrm{g}\right),{ }^{137} \mathrm{Cs}$ $\left(6.8 \times 10^{8} \mathrm{dpm} / \mathrm{g}\right)$, and ${ }^{241} \mathrm{Am}\left(1.4 \times 10^{8}-1.8 \times 10^{8} \mathrm{dpm} / \mathrm{g}\right)$.

- The particle size analysis shows a large fraction of particles greater than $100 \mu$.

\subsection{REFERENCES}

${ }^{1}$ G. D. Thaxton, “Tank 5 Feed and Bleed Residual Solids Sample Analysis”, HLE-TTR-2010-014, Rev. 1, April 27, 2010.

${ }^{2}$ M. R. Poirier, D. DiPrete, and C. Coleman, “Task Technical and Quality Assurance Plan for Analysis of the Tank 5F Feed and Bleed Residual Solids”, SRNL-RP-2010-00729, Rev. 0, April 27, 2010.

${ }^{3}$ M. R. Poirier and S. D. Fink, “Analysis of Samples from Tank 5F Chemical Cleaning”, SRNL-STI-2009-00492, December 9, 2009.

${ }^{4}$ M. S. Hay, K. P. Crapse, S. D. Fink, and J. M. Pareizs, "Characterization and Actual Waste Tests with Tank 5F Samples," WSRC-STI-2007-00192, Washington Savannah River Company (2007). 